\title{
Observation of continuum radiations from the Cluster fleet: first results from direction finding
}

\author{
P. M. E. Décréau ${ }^{1}$, C. Ducoin ${ }^{1,}{ }^{*}$, G. Le Rouzic ${ }^{1}$, O. Randriamboarison ${ }^{1}$, J.-L. Rauch ${ }^{1}$, J.-G. Trotignon ${ }^{1}$, X. Vallières ${ }^{1}$, \\ P. Canu ${ }^{2}$, F. Darrouzet ${ }^{3}$, M. P. Gough ${ }^{4}$, A. M. Buckley ${ }^{4}$, and T. D. Carozzi ${ }^{4}$ \\ ${ }^{1}$ LPCE, CNRS et Université d'Orléans, Orléans, France \\ ${ }^{2}$ CETP, CNRS et Université Versailles St Quentin, Vélizy, France \\ ${ }^{3}$ Belgian Institute for Space Aeronomy, (IASB-BIRA), Brussels, Belgium \\ ${ }^{4}$ Space Science Centre, the University of Sussex, Falmer, Brighton, UK \\ *March-June 2003 visit at LPCE
}

Received: 9 October 2003 - Revised: 6 May 2004 - Accepted: 12 May 2004 - Published: 14 July 2004

Part of Special Issue "Spatio-temporal analysis and multipoint measurements in space"

\begin{abstract}
The Cluster fleet offers the first possibility of comparing non-thermal terrestrial continuum radiation from similarly equipped nearby observation points. A very rich data set has already been acquired on the Cluster polar orbit, between 4 and 19 Earth radii geocentric distances, and preliminary analysis has been carried out on these emissions. We focus in this paper on direction finding performed from all four spacecraft as a means to locate the position of the sources of this continuum radiation. Directions are derived from spin modulation properties, under the usual analysis assumptions of the wave vector of the radiation lying in the plane containing the spin axis and the antenna position at electric field minimum. All the spin axes of the four Cluster spacecraft are aligned perpendicular to the ecliptic, thus the aligned spacecraft antenna spin planes provide redundant 2$\mathrm{D}$ views of the propagation path of the radiation and source location. Convincing 2-D triangulation results have been obtained in the vicinity of the source region. In addition, the out of spin plane component of the wave vector reveals itself to a certain extent through directivity characteristics compared at different distances of the spin plane to the ecliptic. The four case events studied (two of them taken near apogee, the other two near perigee) have confirmed general properties derived from previous observations: trapping in the lower frequency range, radiation escaping into the magnetosheath region in the higher frequency range. All propagation directions are compatible with source positions in the plasmapause region, however, at a significant distance from the equator in one case. Our observations have also revealed new properties, like the importance of small-scale density irregularities in the local amplification of continuum radiation. We conclude that more detailed generation and propagation models are needed to fit the observations.
\end{abstract}

Correspondence to: P. M. E. Décréau

(pdecreau@cnrs-orleans.fr)
Key words. Magnetospheric physics (plasmasphere) Space plasma physics (instruments and techniques, radiation processes)

\section{Introduction}

Continuum radiations are electromagnetic radiations of terrestrial origin, covering the range from a few $\mathrm{kHz}$ to a few $100 \mathrm{kHz}$, often present in the Earth's environment. First discovered about 30 years ago (Gurnett, 1975, and references therein) they have been observed from all geo-spatial missions suitably equipped, in particular on ISEE, GEOS, DE I, GEOTAIL, WIND (Morgan and Gurnett, 1991, and references therein; Kasaba et al., 1998, and references therein). The appearance of continuum radiation in the observations (e.g. as shown in Figs. 1, 3, 5, 6, 7, 8) is quite variable, depending on the point of the observation, on the frequency range examined, on the state of the dynamical magnetosphere, and also on instrument performances. Basic characteristic features are: weak emissions grouped as a series of narrow band frequencies, displaying spectral stability with time (several hours are typical). Variations of that description include smooth emissions over a large frequency "continuum" range, continuum enhancements structures, i.e. sudden onset with rapidly diverging frequency bands of roughly equal separation at any one time, and other more refined descriptions.

The continuum sources are thought to lie mainly in the equatorial plane, in the vicinity of a plasmapause gradient (Gurnett et al., 1988). This does not exclude the possibility of a source location near the magnetopause, which would fit better some observations. In any event, the multiplicity of simultaneous sources seems to be a rule, as well as the multiplicity of ray paths between sources and points of observation, resulting in the various possible continuum forms 
observed. Two main types of generation mechanisms have been proposed: linear and non-linear. Both involve high level electrostatic emissions at or above the upper hybrid frequency. Some generation mechanisms have been discussed in view of observations (Barbosa, 1982; Etcheto et al., 1982; Morgan and Gurnett, 1991). However, the question about which processes are at work remains largely unanswered. More investigations are thus required before a complete and comprehensive understanding of the detailed features and signatures observed is available.

In this paper, our goal is to describe which novel observational features are added by the Cluster fleet (Escoubet et al., 2001) to the already important knowledge available, in particular by direction finding. We shall neither discuss generation mechanisms nor propagation properties which would sustain such observations. But we will try, in the light of the few case events presented, to discuss some relevant questions.

Observations from the Cluster fleet are interesting in several aspects. The main one is certainly the presence of several simultaneous observational points of a given event. Another important aspect is the orbit configuration, which offers views at a close vicinity of sources, or even passing through areas where conversion mechanisms from intense electrostatic waves into electromagnetic emissions are at work. Lastly, the capabilities of the main instrument of observation, the Whisper experiment (Décréau et al., 1997; 2001), lead to a very rich set of observations, specific to Cluster, which can be taken advantage of in a number of ways. This paper aims at presenting a flavour of the quality of the observations, via the example of a few case events. The next section describes the instrument capabilities, and the technique of directivity analysis derived from Whisper data. Section 3 presents low-latitude observations in the tail magnetosphere and in the magnetosheath, obtained in both cases with the largest spacecraft separation (triangulation baseline distance) available until this time. Section 4 focuses on observations in the vicinity of the sources. Section 5 presents a summary and a conclusion.

\section{Whisper instrument measurement capabilities}

\subsection{Main performances}

The Whisper experiment (Décréau et al., 1997) includes two main devices. One is a receiver and Fast Fourier Transform vector processor, which treats the electric field signal provided by the EFW instrument (Gustafsson et al., 1997), and calculates every $13.33 \mathrm{~ms}$ a frequency spectrum in the range $2-80 \mathrm{kHz}$, at a constant frequency separation of 160 or $320 \mathrm{~Hz}$ (480 or 240 frequency bins). It is mainly used in the so-called Natural mode of operation, where several spectra are accumulated, frequency to frequency, and a selection of the resulting averaged spectra (covering a time span of $213 \mathrm{~ms}$, in standard operations), is subsequently transmitted to the ground.
The second part of the instrument is a transmitter, which actively triggers natural resonance in the electronic population. This module, added to the receiver and analyser, forms a sounder (Trotignon et al., 2001). When the instrument operates as a sounder, the central frequency of a short triggering wave train sweeps the $4-80 \mathrm{kHz}$ range step after step. The main output in the so-called Sounding mode, which consists of a frequency spectrum (over the $4-80 \mathrm{kHz}$ band), constructed from the partial frequency responses obtained after each step. The instrument is operated as a sounder during short intervals of about $3 \mathrm{~s}$ (covering two frequency sweeps), at a standard recurrence of $52 \mathrm{~s}$. The rest of the time, it is operated in Natural mode. It then measures electric field variations at a sensitivity better than $2 \cdot 10^{-7} \mathrm{~V}_{r m s} \mathrm{~Hz}^{-1 / 2}$ (converted in units of electric field spectral density, $5 \cdot 10^{-18} \mathrm{~V}_{\mathrm{rms}}$ $\mathrm{m}^{-1} \mathrm{~Hz}^{-1}$ ). Such a sensitivity is well adapted to the measurement of typical low intensity continuum radiation emissions, as will be seen later.

\subsection{Continuum observations by the Whisper instrument: general features}

The main output of the Whisper instrument is best presented as colour-coded frequency-time spectrograms, constructed from the successive frequency spectra elaborated on board, either in Natural or Sounding modes. The presence of continuum radiation is regularly visible in Whisper spectrograms. The top and middle panels of Fig. 1 present observations taken at large distances from the source, close to the ecliptic, during geophysically active periods (Km levels, respectively, at $4^{-}$and $3^{+}$). They look similar to observations measured in similar regions and reported in former studies, for instance, by ISEE (Kurth et al., 1981) or GEOTAIL (Kasaba et al., 1998). In the first case event (upper panel), Cluster 4 spacecraft is located in the magnetosheath, characterized by a local plasma frequency $\mathrm{F}_{p}$ fluctuating between about 30 and $40 \mathrm{kHz}$, and by strong sporadic electrostatic fluctuations at low frequency. The main escaping continuum features, here superposed to type 3 emissions, display a frequency separation of approximately $11 \mathrm{kHz}$, indicative, if measuring the gyrofrequency at the source, of a main source at about $4.2 R_{E}$ geocentric distance (Gough, 1982).

The second case (middle panel) shows an example of a continuum enhancement feature observed by Cluster 4, a phenomenon first reported by Gough (1982), as remote sensing the plasmapause motion. Here, the frequency separation in the main "finger-like" feature after 23:00 UT amounts to about $14.5 \mathrm{kHz}$, indicative of a corresponding source at a geocentric distance of about 3.9 Earth radii.

The bottom panel of Fig. 1 shows a spectrogram measured onboard Cluster 2 under moderately disturbed conditions ( $\mathrm{Km}$ level at $2^{\circ}$ in the plasmasphere region). It can be compared to Dynamic Explorer 1 (DE 1) views (see Morgan and Gurnett, 1991, plates 1 and 4), because orbit paths are locally similar (perigee of Cluster roughly corresponds to apogee for DE 1). Whisper offers additional information, however: the sounder's resonance patterns, displayed 

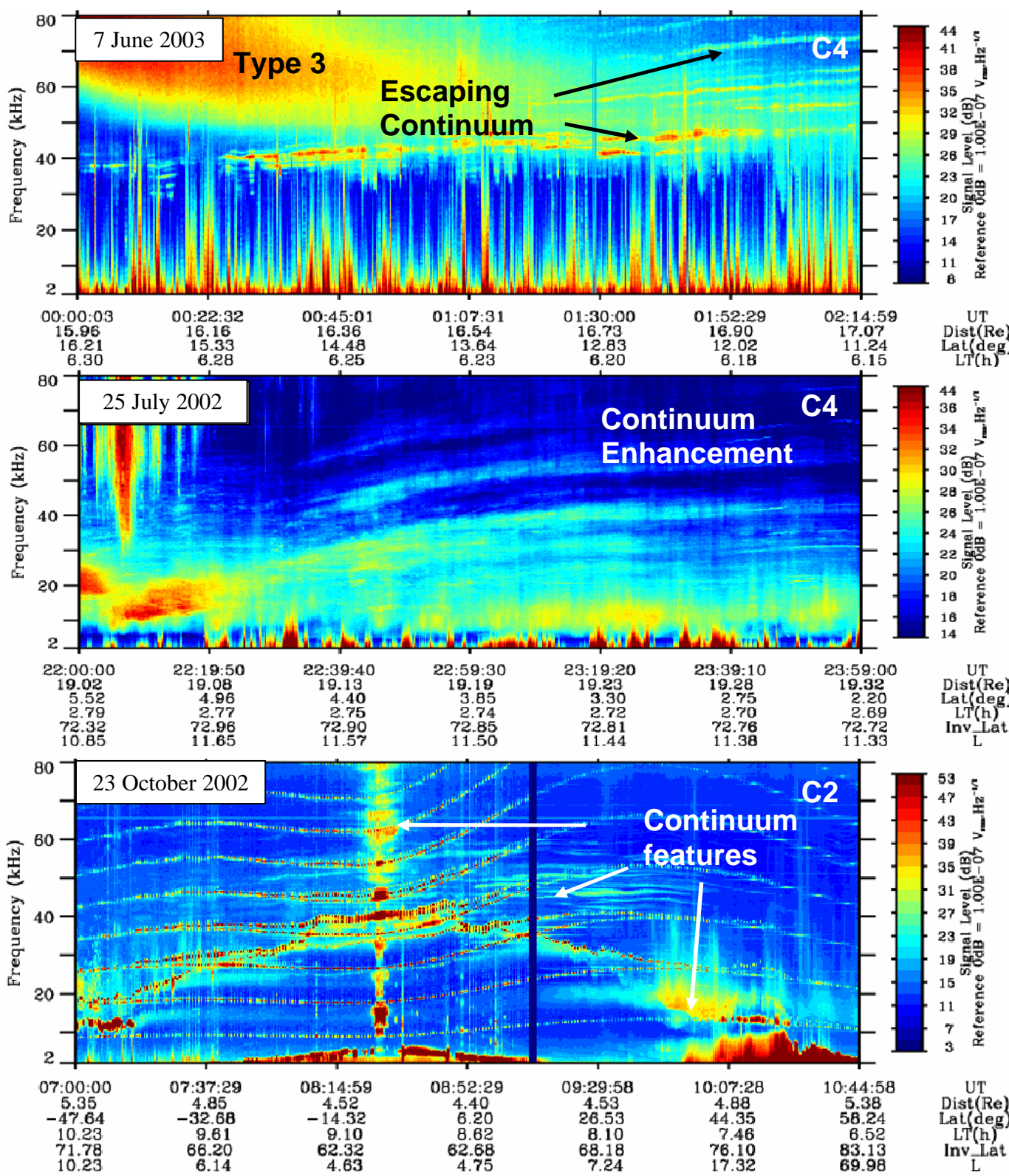

Fig. 1. Frequency-time spectrograms showing continuum emissions measured by Whisper instrument on three different days. The two upper panels display natural emissions only, while the lower panel also includes the resonance spectral harmonic lines induced during sounding operations. Continuum radiations appear under different forms: in the 7 June 2003 case, as a series of narrow frequency elements, penetrating the magnetosheath, in the 25 July 2002 case, as a continuum enhancement, starting simultaneously with auroral radiations signatures, both likely resulting from the same triggering due to particle injection and precipitation, in the 23 October 2002 case, either above $40 \mathrm{kHz}$, as structured emissions localized near the magnetic equator, or between 30 and $50 \mathrm{kHz}$, as narrow frequency continuum elements, or else at low frequency, as a smooth large band emission, trapped in between the plasmaspheric body and a density blob.

here superposed on natural emissions. The spacecraft, located initially in the Southern Hemisphere, travels inward, traverses the body of the plasmasphere near perigee, and then progresses on its outer leg. The resonance pattern points in particular to the local electron gyrofrequency $(\sim 8-12 \mathrm{kHz})$ and harmonics, $\mathrm{n}_{c e}$, the plasma frequency, $\mathrm{F}_{p}$, ( increasing from about 10 to about $32 \mathrm{kHz}$ inside the plasmasphere, then decreasing), and the $\mathrm{F}_{q}$ Bernstein series (see Trotignon et al., 2001, for a description of the sounder triggered res- onance patterns). The magnetic equator is crossed at about 08:30 UT, as indicated both by the vicinity of a minimum in $\mathrm{F}_{c e}$ variations with time and by the presence of high intensity electrostatic wave peaks, distributed predominantly between the harmonics of local electron gyrofrequency (Gough et al., 1979). The perigee proper is reached at about 08:50 UT. Such a region of observation is particularly interesting, because it is where continuum generation is expected to occur. Three different forms of continuum are co-existing in this 
particular case, as indicated on the plot: (i) features confined to the near vicinity of the equator, mainly above $45 \mathrm{kHz}$, (ii) longer lasting narrow frequency lines in the $30-50 \mathrm{kHz}$ bandwidth, similar to escaping continuum features shown in the top panel (here fading above $\sim 45^{\circ}$ magnetic latitude), (iii) unstructured emissions in the $10-25 \mathrm{kHz}$ bandwidth, similar to low frequency features shown in the middle panel. The first type might be the primitive form of continuum radiation. It is seen at close proximity to the equatorial high intensity electrostatic emissions thought to be converted to electromagnetic radiation. The characteristic frequencies of equatorial emissions can be identified using the sounder's operations. Indeed, the capability of the Whisper instrument to indicate local properties of the medium (Trotignon et al., 2003; Canu et al., 2001), together with a high level of detail in the spectra, is extremely interesting for a study of generation mechanisms. In particular, each wave spectrum delivered might be considered as instantaneous: it compares electric field intensity at different frequencies for the same acquisition in time and with a frequency resolution sufficient to separate rays only $1 \mathrm{kHz}$ apart, even in the higher frequency range. Hence, levels at the induced Bernstein $\mathrm{F}_{q} \mathrm{~s}$, and at the $\mathrm{F}_{p}$ or the upper hybrid $\mathrm{F}_{u h}$ natural emissions are directly comparable. It can be seen, for instance, that in this case the most intense natural electrostatic emissions at the magnetic equator do not occur in the electron cyclotron harmonic band containing the $\mathrm{F}_{u h}$ frequency (located in this case at $\sim 32 \mathrm{kHz}$ in the third band). Instead, they appear in the vicinity of the $\mathrm{F}_{q 4}$ and $\mathrm{F}_{q 5}$ frequencies ( 40 and $46 \mathrm{kHz}$ ), at an intensity at least one order of magnitude higher than the intensity observed at $\mathrm{F}_{u h}$. A complete discussion of those observations is outside the scope of this paper. However, such a behaviour as multiple driving energy sources at a given point might partly explain the multiplicity of continuum lines observed, whose characteristic frequency separation is much smaller than the local gyrofrequency (see as an example the pattern around 09:30 UT, in the $30-50 \mathrm{kHz}$ frequency range).

\subsection{Direction finding analysis}

Continuum radiation, when measured from a spinning antenna, is modulated at twice the spin frequency, a property which has been used in the past to derive ray path direction in the spin plane (Gurnett, 1975; Filbert and Kellogg, 1989; Morgan and Gurnett, 1991; Kasaba et al., 1998). All spin planes of the Cluster spacecraft are parallel to the ecliptic; we have followed a standard approach to derive the propagation direction projected in this plane. The wave is assumed to propagate in free space, as a planar wave. Its electric field is supposed to be either randomly or circularly polarized. Under such assumptions, the electric field pattern of the wave will project into the spin plane as a polarization ellipse, the minor axis of which is aligned with the wave vector $\boldsymbol{k}$ of the radiation projected in to the same plane. In order to quantitatively locate the latter direction, we define the angle $a k$ which the projected wave vector makes with the X GSE-axis, as illustrated in the upper sketch of Fig. 2. The value of $a k$ is estimated in the $0-180^{\circ}$ range, not by a simple detection of the antenna attitude at minimum (or maximum) intensity, but by a fit of the complete measured spin modulation to an abs(cosine) shape. The modulation index $m$ derived through this process is $m=\cos (\psi)$ for a point source, where $\psi$ is the angle of the radiation ray with respect to the spin plane.

The $m$ index depends in addition upon the dimension of the source. It decreases when either one of the following conditions, a propagation vector close to the spin plane or a source of small dimension, is not satisfied. The direction finding analysis provides thus results easier to interpret when $\psi$ is small and hence one of these conditions is well defined. If sources are placed in the equatorial plane, the small $\psi$ condition is expected to be well satisfied only on part of the Cluster polar orbit, i.e. in the vicinity of either perigee or apogee, which are close to equator. We indeed obtain statistically (over a selection of continuum events) higher modulations when Cluster is placed at low latitudes, in the vicinity of either perigee or apogee, than elsewhere. Moreover, a set of observations (Morgan and Gurnett, 1991; Green and Boardsen, 1999) indicate that continuum sources are emitting within a cone angle limited in latitude. As a consequence, only part of the Cluster orbit, again near perigee or apogee, should be illuminated. Surprisingly, we still obtain, from our limited statistical study, continuum events at rather large latitudes (up to $60^{\circ}$ and more), most characterized with a low modulation index $m$, but some with a significant ( $>0.5) m$ value. It is worth noting, however, that "large" latitude conditions associated with a significant modulation of continuum radiations are met on the perigee side of the Cluster orbit, at small geocentric distances $\left(<6 R_{E}\right)$. Hence, although technically observed at high latitude, those radiations can still travel at a reasonably small angle with the spin plane: on the one hand, they might emit from a source being placed at a few Earth radii off the equator, on the other hand, they might have undergone one reflection at magnetopause and travel at high latitudes (above the Earth), as shown in ray-tracing calculations of Green and Boardsen (1999). The details of the direction finding analysis are constrained by the characteristics and performances of the Whisper instrument. Coping with the limited telemetry available, on-board compression reduces the size of each frequency bin spectral density value to a 6-bit word, leading to some granularity in the intensity information (see Décréau et al., 1997). In addition, the time resolution is such that only a few spectra at most are delivered per 4-s spin period (standard resolution: $2.15 \mathrm{~s}$, best resolution: $0.33 \mathrm{~s}$ ). Three main parameters are derived after fitting the data with an abs(cosine) model: the directivity angle $a k$, the amplitude of the modulated signal and the modulation index $m$. In addition, we calculate the standard deviation between the experimental and the modelled signal values and validate a fit only if the signal to standard deviation ratio is better than $6 \mathrm{~dB}$, and if the modulated signal is higher than the electronic noise threshold. Lastly, the modulation index value is re-evaluated by applying a correction factor. This is needed because each experimental point derives from a spectrum obtained by accumulation of 


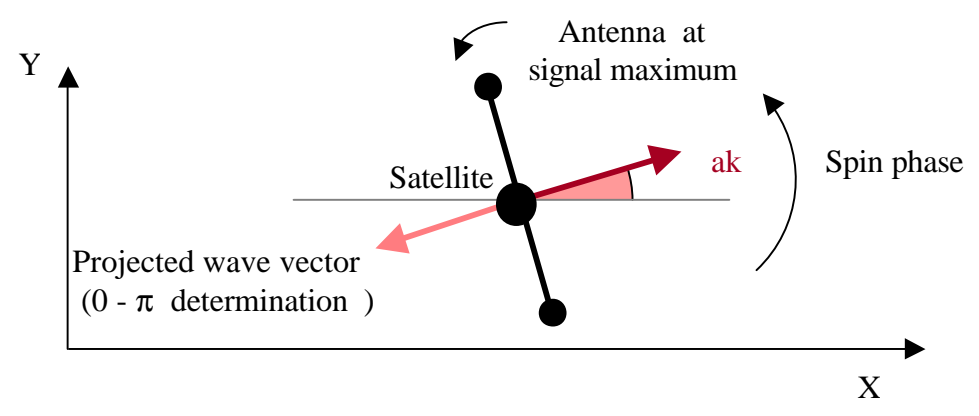

$20021023 \quad 09: 37: 30 \quad 30.0 s \quad f=46.2 \mathrm{kHz}$
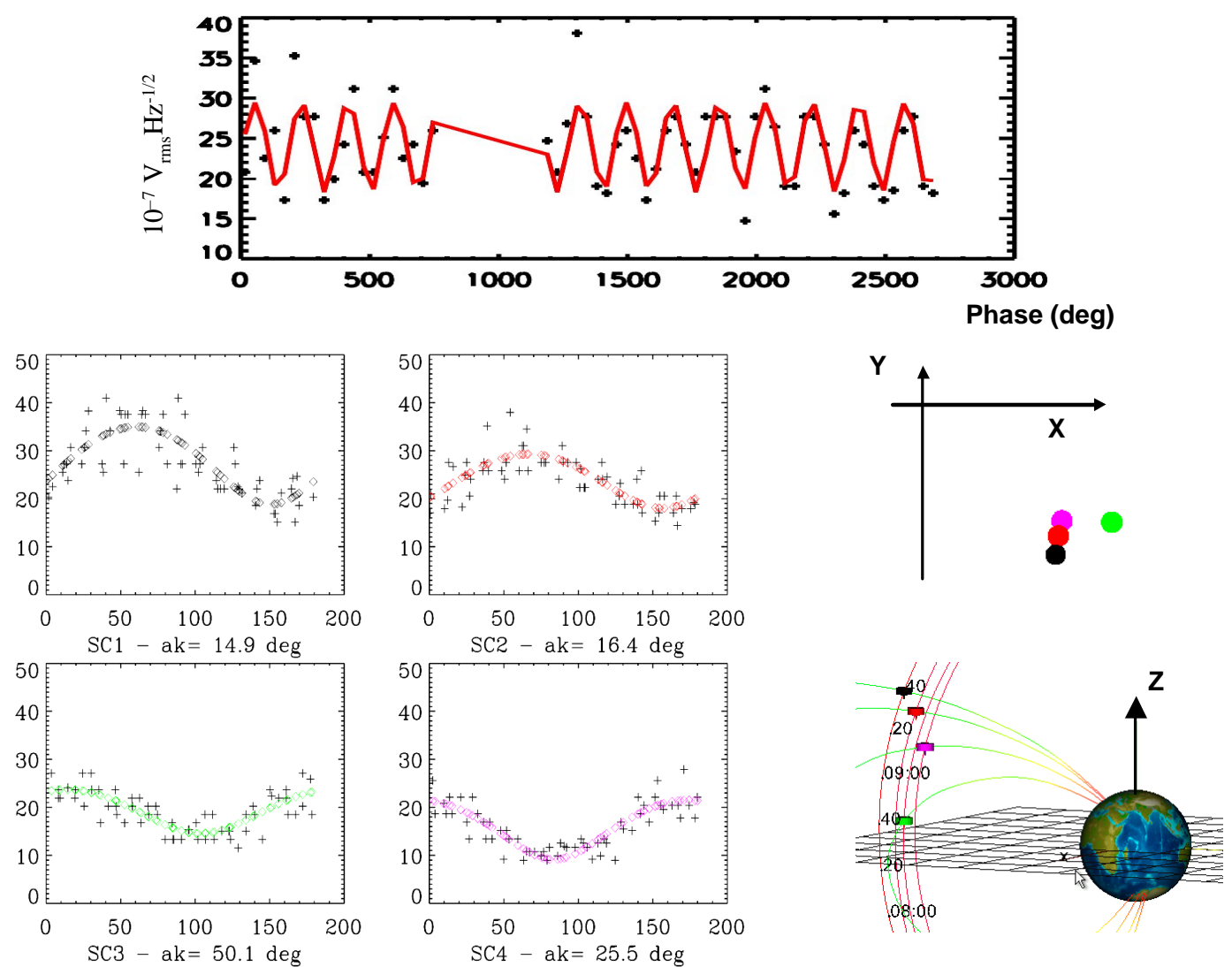

Fig. 2. Derivation of $\boldsymbol{k}$ vector orientation in the XY GSE-plane. Top panel sketch of the antenna in the spin plane defining the directivity angle in 2-D. Second panel from top: signal intensity (expressed in units of normalized voltage difference) versus time given as phase angle $\omega t$ (expressed in degrees). Experimental values are plotted as crosses, the modelled variation is plotted in red. Four bottom left panels: signal intensity values (same unit as above) measured on the four spacecraft (same time and frequency as above), versus modelled intensity variations (phase angles restricted to $0-180^{\circ}$ range; colours according to standard conventions on Cluster, black, red, green and purple for, respectively, C1, C2, C3 and C4). Two bottom right panels: Cluster configuration in OVT representation (Stasiewicz, 2001), using the GSE reference system: projections in the XY-planes (top) and YZ-planes (bottom).

successive individual spectra. During the accumulation, the antenna rotates, sometimes from an angle as high as $80^{\circ}$. We link the corresponding accumulated spectrum to the phase angle at half rotation, which still samples the spin plane adequately, but the modulation index is under-evaluated, from below $2 \%$ under standard operations, to $7 \%$ in the case of Fig. 2, or 30\% in the worst case. The choice of the time interval duration for analysis depends strongly on the operation mode. Tests have demonstrated that the most stringent factor towards obtaining a satisfying fit is the signal stability. We have thus favoured a frequent analysis, relying upon about 30 to 40 experimental points ( 30 to 40 accumulated spectra). The corresponding duration is $90 \mathrm{~s}$ in the standard operational mode, less in burst telemetry mode (as is the case of Fig. 2). On the other hand, we currently carry out the analysis over a single frequency line (which covers a frequency band 
of either 160 or $320 \mathrm{~Hz}$ ) in the available spectra. However, we have also the facility to carry the analysis over the highest intensity line in a small frequency band, which proved useful in some cases.

The plots in Fig. 2 (middle and bottom left panels) illustrate the quality of direction analysis obtained with Whisper data during the third event shown in Fig. 1. The analysis is done on one element of escaping continuum $(46.2 \mathrm{kHz}$, 09:37 UT). The Cluster instruments are running in burst mode, which allows a fare time resolution $(0.65 \mathrm{~s})$. In the middle panel, the amplitude intensity is plotted against the antenna rotation angle for $\mathrm{C} 2$ while the bottom panel displays measured intensity variations for each spacecraft against the phase angle restricted to the $0-180^{\circ}$ range. Modelled variations are superposed in both cases. The middle panel shows the details of time evolution. Fifteen half rotations are accomplished within the 30-s time sample, about two of those are devoted to sounding operation (between 700 and $1200^{\circ}$ rotation phase angle), which interrupts the natural emission survey. That type of plot is useful to depict unwanted spurious bursts of signal enhancements. Actually, we apply a modest filtering (suppressing the widely out of range values) to our data set prior to proceeding with data fit. This explains the small differences between the middle and bottom panel views for $\mathrm{C} 2$.

It is important to estimate uncertainty factors on directivity parameters. Indeed, making the best use of four spacecraft is much more demanding than dealing with measurements from a single platform, where all the fluctuations observed could possibly be attributed to time fluctuations in the source. All former studies demonstrated that the fluctuations are important (see, for instance, Kasaba et al., 1998), and the Cluster measurements do not contradict that fact. However, as shown later, the Cluster data indicate that the effects from granulation in space are another important factor which limits triangulation possibilities toward finding the source location.

The antenna spin angle is known with a good accuracy, better than a degree, and this uncertainty factor can be neglected relative to what results from random noise contained in the electric signal. Individually, each propagation vector angle position is determined with an uncertainty which can be estimated from the standard deviation between the modelled and the experimental spin modulation. A simple relation between this quantity and an angle shift in the modelled modulation leads to averaged angle deviations, which depend significantly on the instrument operational mode and on the plasma regime encountered. In the case illustrated in Fig. 2, it amounts to less than $\pm 1^{\circ}$, and can be translated to a confidence (uncertainty) interval better than $\pm 2^{\circ}$. In that particular case, each experimental point results from averaging 32 individual spectra, which reduces local fluctuations. Under standard conditions, only 16 individual spectra are averaged, and, in addition, the necessary time interval for analysis is more important ( $90 \mathrm{~s}$, as compared to $30 \mathrm{~s})$. This results in estimated angle uncertainties of, typically, $\pm 7^{\circ}$.

In the example of Fig. 2, Cluster satellite $\mathrm{C} 3$ locates the propagation direction of the fine frequency line analysed at about $30^{\circ}$ from the directions estimated by the three other spacecraft. Such a difference persists on successive directivity estimations. That particular case is interesting, because it appears that all directions do not point to a same source region. The satellite $\mathrm{C} 3$ position differs mainly from the other spacecraft positions by its latitude in the GSE frame: about $15^{\circ}$ as compared to about $35^{\circ}$ (see sketch in Fig. 2, bottom right). A complete analysis of this event is out of the scope of this paper, but it is worth noting that the Cluster platforms regularly detect important differences between propagation directions, when travelling near perigee.

\section{Low-latitude observations at large distance from the source}

\subsection{Case of 25 July 2002: nightside magnetosphere}

We present here the results of directivity analysis during the continuum enhancement event shown in Fig. 1 (middle panel). The four spacecraft are located in the nightside magnetosphere (at LT about 3), close to apogee, at small $\left(<5^{\circ}\right)$ latitude. They are separated at most by $0.8 R_{E}$ in the XYplane. The local plasma frequency can be approximated from the low-frequency cut off on the continuum emissions spectra, located below $10 \mathrm{kHz}$ (see Fig. 1, bottom). The two upper panels in Fig. 3 display, in the form of frequency/time spectrograms, the modulation factor $m$ and the directivity angle $a k$, respectively, calculated for a two-hour time interval. In this particular case, taken under normal telemetry, the frequency separation in the Whisper spectra is the best possible, $160 \mathrm{~Hz}$, each spectrum results from the accumulation of 16 individual spectra (covering $213 \mathrm{~ms}$ ), and the time resolution is $0.85 \mathrm{~s}$ (one out of four accumulated spectra is downloaded). We use 2-min running time intervals to derive directivity parameters. We limit the analysis to frequencies higher than $10 \mathrm{kHz}$, largely above the local plasma frequency, which allows us not to subtract the level of quasi-thermal noise as would be otherwise necessary (Steinberg et al., 1990). The white background indicates that the criteria for successful direction finding (see Sect. 2.3) are not met and hence the analysis is not applicable. This is the case, for example, for the intense high frequency auroral emissions occurring above $60 \mathrm{kHz}$ at the beginning of the time interval shown in Fig. 1. These emissions, although directional, are too sporadic for the spin modulation method to be applicable. Several interesting features are showing up in the spectrograms of Fig. 3:

1. In the second panel three main diverging frequency bands are seen at $a k$ angles in the range $30-70^{\circ}$ (greenish colour code), contrasting with the background emissions at $a k$ angles close to zero (blue or black colour code),

2. In the top panel, modulation factors above $90 \%$ are linked with the two upper rising frequencies, less contaminated by background signal, at a frequency separation of $\sim 14.5 \mathrm{kHz}$. A gradient in modulation index 
(decreasing from $>60 \%$ to $<40 \%$ ) is observed at a transition frequency varying in the $20-35 \mathrm{kHz}$ range. Such a transition has been recently used as a signature of the upper frequency of trapped continuum, yielding a remote information, when observed in the far tail, about plasma frequency in the magnetosheath flanks (Nagano et al., 2003),

3. Before 22:25 UT, at highest radiation intensity (see Fig. 1), all $a k$ angles show Sun-Earth aligned propagations (shown as blue), even in the frequency ranges (30$50 \mathrm{kHz}$ ), where later they turn to a different spacecraftEarth, direction (shown as green). Due to the $180^{\circ}$ ambiguity in direction finding, we cannot tell which are the actual source positions. We will go back to this point later, when discussing possible scenarios (Fig. 4).

4. At low frequency (below $15-25 \mathrm{kHz}$ ), although the radiation intensity is significant (see Fig. 1 around 23:20 UT, for instance), the direction finding method fails. The modulation index decreases thus below the acceptance level ( $\sim 20 \%$ in that case), or the signal becomes too noisy.

A simple interpretation of those features, suggested by the pioneering work of Gurnett (1975) and confirmed later by others, is the following: the radiation of lower frequency, below $15-25 \mathrm{kHz}$, is probably trapped after multiple reflections at the magnetopause, losing its directional character; the first episode (before 22:25 UT) at higher $(25-50 \mathrm{kHz})$ frequencies indicates a radiating source, S1 say, of rather large dimension (suggested by the moderate modulation factor) at a smooth plasmapause profile (as suggested by the large frequency range), the overall beam being collimated along the Sun-Earth direction; in the second episode (after 22:25 UT) a source, S2 say, appears. It is confined to a sharp plasmapause gradient (as suggested by the frequency pattern, characteristic of continuum radiation from a source at a well-defined gyrofrequency) radiating from a small area (as suggested by the high modulation factor) in a cone angle off the Sun-Earth direction. The apparition of S2 can be accounted for either a temporal evolution (it switches on) or a spatial evolution (it comes in the line of sight). We will come back to this point later.

The bottom panels of Fig. 3 present some statistics of the calculated angle values shown in the middle panel for the satellite $\mathrm{C} 4$, allowing in particular a comparison between spacecraft. For three selected time intervals of $10 \mathrm{~min}$ the panels show histograms of the $a k$ values in the same $20-70 \mathrm{kHz}$ frequency range. That frequency range is wide, and the derived information should be taken as complementary to detailed instantaneous frequency dependence of source-spacecraft direction, illustrated in the upper panel of Fig. 4. The curves presented at the top of Fig. 4 display a repetitive pattern, peaking approximately at half harmonics of $14.5 \mathrm{kHz}$, but showing details such as plateaus, secondary peaks, which are consistently similar on all four spacecraft; (the plots, colour coded in the standard way, being here su-
WHISPER 1 25/07/2002
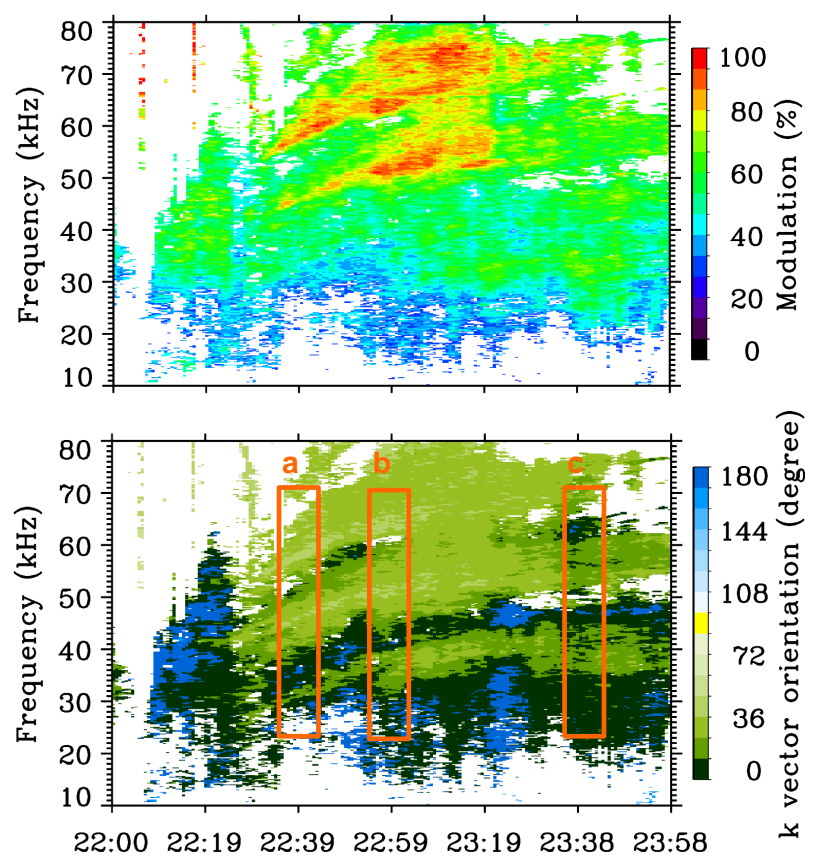

Universal Time
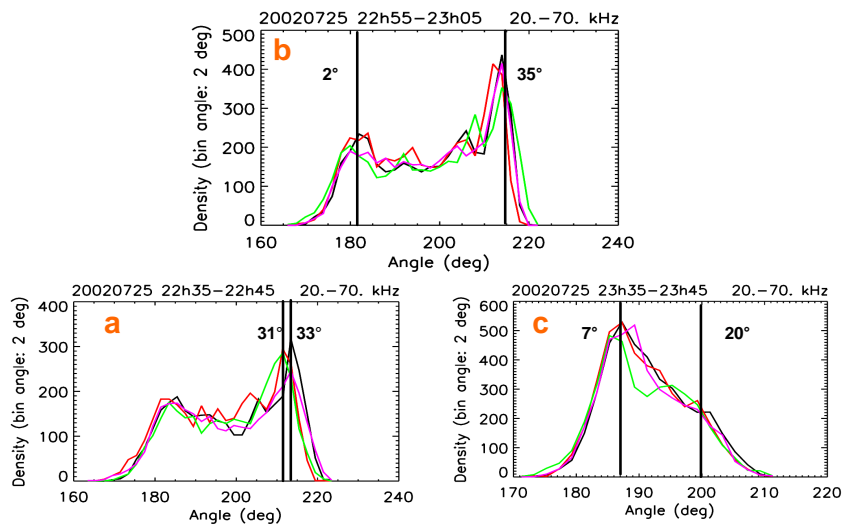

Fig. 3. Direction analysis for the 25 July event (middle panel of Fig. 1). Two upper panels: frequency/ time spectrograms of the directivity parameters calculated for $\mathrm{C} 1$ (same time interval as dynamic spectrogram presented in Fig. 1); top panel: modulation index; middle panel: $a k$ direction angle values. Three lower panels: histograms of the $a k$ angle values obtained with the four spacecraft data sets over three frequency/time intervals (marked above as intervals $\mathrm{a}, \mathrm{b}$ and $\mathrm{c}$ ). The standard colour code (described in Fig. 2) allows one to distinguish between the satellites. Results are very similar from spacecraft to spacecraft. Two main propagation directions point out in the core of the event (intervals a and b).

perposed one on each other). Although an interesting subject, we do not investigate nor discuss here in more detail the time and frequency dependence of source directions.

Going back to the histograms displayed at the bottom of Fig. 3, we can nevertheless point to interesting global features. The histograms show that 1) the angular distributions are remarkably similar from spacecraft to spacecraft (we shall discuss later the small difference indicated at left), 
20020725 23:01:00 UT 1: $42.0 \mathrm{kHZ} \quad$ 2: $50.6 \mathrm{kHz}$
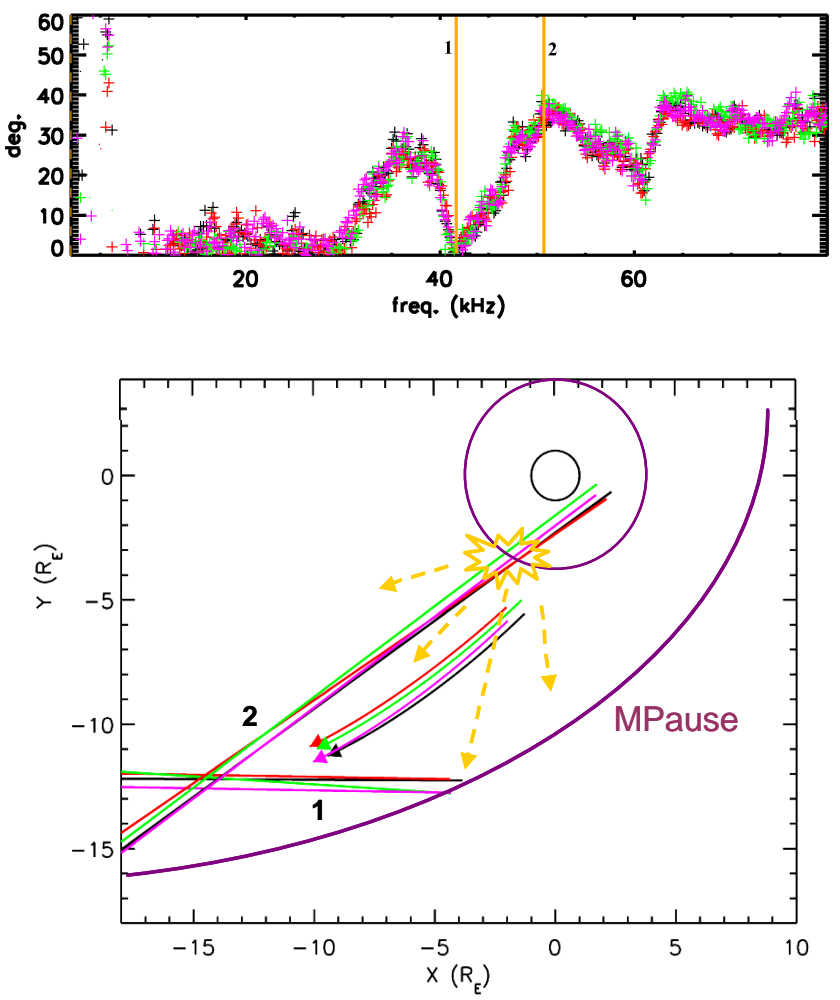

Fig. 4. Direction finding from analysis of a 2-min time interval at about 23:00 UT, in the central part of the 25 July 2002 event. Top panel: detailed variations of the angle $a k$ versus emission frequency. Two frequencies, at two extremes of $a k$ values, are noted, respectively, by labels 1 and 2. Bottom panel: plots of the propagation directions in the XY-plane at the two frequencies selected above. Each spacecraft position is located at the intersection of the two direction lines in the same standard colour. Part of the orbit prior to the event is shown with colour-coded curves ended by arrows.

2) they provide precise angle values at two peaks in the distribution associated with the sources $\mathrm{S} 1$ and $\mathrm{S} 2$, as described above, together with statistical estimation of the related confidence intervals (a few degrees), 3) the angular position of the second source, $S 2$, peaks at the same value $\left(\sim 33^{\circ}\right)$ around 22:40 UT (left panel) and around 23:00 UT (middle panel), although its characteristic frequencies have increased from several $\mathrm{kHz}$, (4) the two main peaking directions bound a cone angle of $\sim 35^{\circ}$ width, where the illumination, as measured by the density of points in a given bin angle, is significant; the number of measured radiating directions falls abruptly to zero outside that cone. Angle value estimations falling inside the cone angle probably result from waves coming from multiple directions, hence should be taken with caution. We have performed a source localisation search by using the two main directions bounding the illuminated cone angle.

From the top panel of Fig. 4, we selected two specific frequencies $(42 \mathrm{kHz}$ and $51 \mathrm{kHz})$ corresponding, respectively, to a minimum and a maximum in the $a k$ angle variations with frequency. Those $a k$ angles are part of the two peaks showing up in the histogram plotted in the bottom panel of Fig. 3, respectively, around 2 and $33^{\circ}$. The bottom panel of Fig. 4 displays the two sets of four propagation directions in the XY-plane as measured by the satellites at the two selected frequencies. We think they are associated with the two sources S1 and S2, which seem to dominate during the whole event, as described above. The directions at the higher $a k$ angle (associated to source S2, label 2) point to the Earth (or opposite to it). If we add the information about the source geocentric distance, as indicated in Sect. 2.2, $\sim 4 R_{E}$ derived from the $\sim 14.5 \mathrm{kHz}$ frequency separation, the continuum ray paths meet the source geocentric distance at $\sim 5 \mathrm{LT}$, which leads to the sketched S2 position (yellow patch), consistent with the classical view of a source located at the plasmapause in the dawn sector. Including this additional information resolves thus the $180^{\circ}$ direction ambiguity in a convincing way.

The directions at low $a k$ values (labeled 1) are more difficult to interpret, as they do not point toward the Earth but toward the dayside magnetopause (or indicating a source in the night sector). The associated modulation index, about $60 \%$, is significant. At this stage, we can only tell that the radiation is probably reflected once in the vicinity of the nose magnetosphere, under such conditions that the collimation is important over a large frequency range, but we cannot say where the primary sources are located. They might be collocated with the source S2. Indeed, such an interpretation is supported by sketching a paraboloid magnetopause at the distance where, acting as a mirror, it would reflect ray paths coming from S2 toward the observed measured direction. The derived magnetopause position is perfectly consistent with the realistic modelled magnetopause used by Green and Boardsen (1999) in their ray-tracing calculations of quasitrapped continuum radiation (see, in particular, Fig. 2 of their paper). Such a scenario would imply that the same restricted source volume radiates over a range of directions. Should the plasmapause surface be smooth, that would rule out a generating mechanism commonly considered for continuum radiation (Horne, 1989): the linear mode conversion "window" theory of Jones (1982). Indeed, according to Jones' theory, the source radiates along a density gradient (oriented perpendicularly to the plasmapause surface), where the conversion efficiency to electromagnetic waves is expected to be high. Rather than ruling out this theory, we think that the perspective of an undulated plasmapause surface, associated with a range of density gradient directions, is quite acceptable. The existence of variable density gradient directions within a small volume in the plasmapause region has recently received direct confirmation by Cluster observations (Darrouzet et al., 2004).

The model of two main sources, S1 and S2, is a way to simplify the presentation. Indeed, we measure ray directions at intermediate $a k$ values, covering a large cone angle. Those intermediate values could be explained by sources positioned along the plasmapause, inside the cone angle, radiating either directly to the spacecraft, or after reflection at magnetopause. 
Whatever the spatial distribution of sources, it is abruptly bounded westward of the global radiation source, which seems to indicate a spatial (in azimuth) boundary. Coming back to the alternative between a temporal and a spatial evolution in the region source at 22:25 UT (when the change in directivity characteristics occurs, and the source $\mathrm{S} 2$ appears), it is difficult to conclude. At this stage, Cluster progresses slowly on its orbit, westward. Less than $0.1 R_{E}$ are covered during the $\sim 10$-minutes duration of the change. That corresponds to $0.3^{\circ}$ in angular dimension, at the considered source to the spacecraft distance. The largest triangulation base, $\mathrm{C} 1$ to $\mathrm{C} 2(\sim 5200 \mathrm{~km})$, yields an angular dimension of $\sim 3^{\circ}$, one order of magnitude higher. If the constellation were entering an intense emission cone issued from the S2 source that would be on its eastward edge - one would expect that $\mathrm{C} 2$ observes $\mathrm{S} 2$ signatures before $\mathrm{C} 1$ does. A detailed examination of the Whisper spectra does not indicate that it is the case. We think that a dynamic evolution of the source is likely at stake, rather than Cluster entering by its own movement in a radiation cone. It could be a fast variation of the position of such a cone (for instance, induced by a modification in plasmapause surface orientation) or a sudden change in the intensity radiated.

As a last remark, let's discuss triangulation capabilities offered by Cluster in such a case (observatories at a distance of about $18 R_{E}$ from the source). The largest triangulation base ( $\mathrm{C} 1$ to $\mathrm{C} 2$ ) leads, when pointing to a small source, to an angular difference $\sim 3^{\circ}$ between the two directions. Such an angular difference is not measurable with Whisper performances, even after averaging data; however, the trend indicated in the bottom left panel of Fig. 3 is of the correct amount $\left(2^{\circ}\right)$ and in the correct direction (smaller angle measured by $\mathrm{C} 1$ than by $\mathrm{C} 2$ ). We may consequently hope for real triangulation possibilities, even close to apogee, if the configuration is stretched to large separations $(15000 \mathrm{~km}$ are considered toward the end of the Cluster mission).

\subsection{Case of 7 June 2003: magnetosheath}

We concentrate here on the event first shown in upper panel of Fig 1. The top panel of Fig. 5 presents a more detailed dynamic spectrogram of this case. Continuum emissions escape the magnetospheric cavity, and are observed above the local magnetosheath plasma frequency. In contrast with the preceding case, several discrete fine structures in frequency are clearly visible, a behaviour commonly observed in the higher frequency range of continuum radiations, where trapping is inefficient. In this particular case, the frequency bin separation of the spectra is $320 \mathrm{~Hz}$ and the continuum elements are probably more narrowly structured than instrument resolution allows one to observe. In addition, intensity enhancements are observed on the lower frequency lines (41 and $47 \mathrm{kHz}$ at 01:47 UT), in conjunction with local density depletions. Density holes are identified by variations in the plasma frequency position $\mathrm{F}_{p}$, which we estimate from the low frequency cut off (pointed by a white arrow) in the smooth electromagnetic radiations observed at that time. They confirm that the magnetosheath characteristics play a role in the propagation conditions of the escaping continuum radiation, and probably of its reflected component. Detailed understanding of the reflection mechanisms and their efficiency could certainly benefit from a study making use of the multipoint Cluster facility.

We have performed direction finding analysis on the bright continuum line running from about 68 to $73 \mathrm{kHz}$ at the top Fig. 5, at two successive times, $t_{1} \sim 01: 45$ UT and $t_{2} \sim 02: 30$ UT. The derived propagation directions are displayed in the bottom panel, in the (spin) plane which is parallel to XY GSE-plane. The 2-D sketch shows in addition the location of the Earth and part of the Cluster orbit projected onto that plane (with colour-coded curves ended by arrows). The four directions are marked, respectively, by labels 1 at $t_{1}$ and labels 2 at $t_{2}$. The total angular dispersion is $\sim 10^{\circ}$ at $t_{1}$, larger than at $t_{2}\left(\sim 6^{\circ}\right)$, perhaps due to the presence at an earlier time of type 3 radiations. In any event, the uncertainty in direction finding, about $\pm 7^{\circ}$ under the standard operations conditions prevailing here, combined with the small spacecraft separation (less than $1 R_{E}$ ), does not allow for any valid triangulation of a source farther than a few Earth radii from the Cluster fleet.

However, if we consider that the frequency separation of the brightest pattern (indicated by yellow arrows in Fig. 5) is the electron gyrofrequency at the source, we can follow the approach used for the preceding event. The electron gyrofrequency value defines the geocentric distance at $4.2 R_{E}$ (see Sect. 2.2). We can then take advantage of the multipoint basis of the Cluster observatory to locate the source as follows: at 01:45 UT, spacecraft 1 and 4, close to each other in the $\mathrm{XY}$-plane, indicate directions $4^{\circ}$ apart. Combined with uncertainty values estimated to about $\pm 7^{\circ}$ on each determination, this provides the $10^{\circ}$ angle span within which the source could be located. The pair 2 and 3, also placed nearby, indicate directions $7^{\circ}$ apart. Combined with uncertainty values, it similarly provides a common angle span of $7^{\circ}$. Finally, both angle spans overlap a common region which crosses the $4.2 R_{E}$ geocentric distance over a local time sector about $3 \mathrm{~h}$ wide, centred at 10:30 LT. That sector, sketched in grey colour Fig. 5 (bottom panel), is actually close to the intersection of directions for spacecraft 1 and 3. Later, at 02:30 UT, the direction finding results show a tendency for the source to be located closer to the Earth, but without any strong shift in local time.

The derived location of the source, based on straight propagation, is compatible with the average position of the plasmapause. This indicates that refraction at the magnetopause boundary is not a phenomenon of crucial importance at the frequency considered. However, owing to the large density fluctuations observed locally in the magnetosheath, some amount of ray curvature along the source to spacecraft path is not excluded. In addition, the derived source to spacecraft direction, almost tangential to a modelled "smooth" plasmapause surface, raises again the question of local plasmapause orientation associated to Jones's theory. To analyse further this event, we have conducted a 

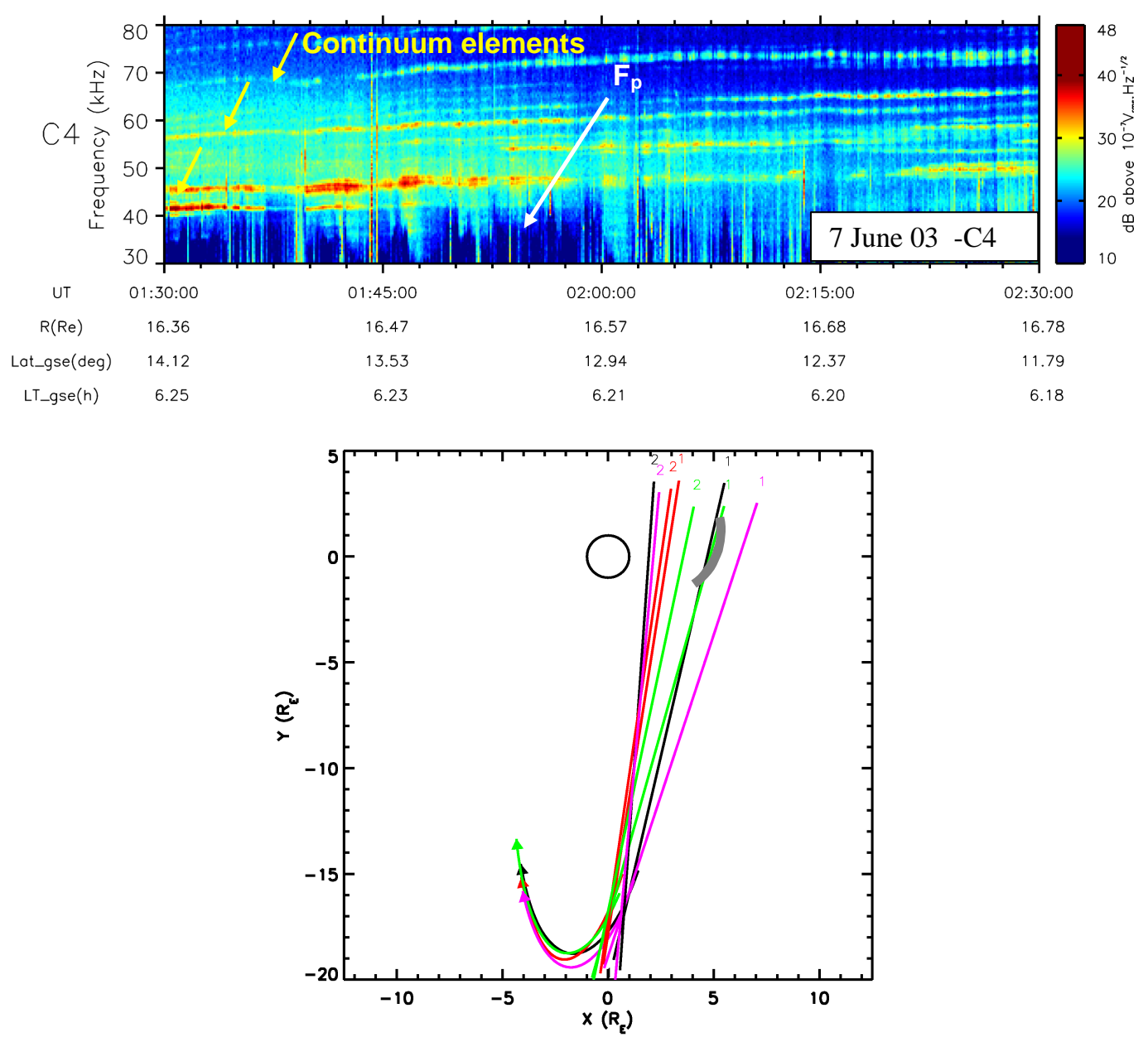

Fig. 5. Direction analysis for the 7 June 2003 event (top panel on Fig. 1). Top panel: details of a frequency-time spectrogram showing continuum elements observed in the magnetosheath. The frequency separation of the three main emission line elements (indicated with yellow arrows) is approximately $11 \mathrm{kHz}$. The position of local plasma frequency is indicated by a white arrow (low frequency cut off in type 3 emissions). Bottom panel: propagation directions observed from the four spacecraft in the spin plane, close to the XY GSE-plane. A distinct single emission line -the upper of the three indicated elements- has been selected for analysis. Its frequency increases slightly from $71.0 \mathrm{kHz}$ at $\sim 01: 45 \mathrm{UT}$, to $73.2 \mathrm{kHz}$ at $\sim 02: 30 \mathrm{UT}$. The directivity angles are calculated over the same $90 \mathrm{~s}$ time interval on each spacecraft, first at 01:45 UT (labels 1), then at 02:30 UT (labels 2). The propagation lines are plotted according to standard spacecraft colour codes (see Fig. 2 caption). The estimated source position at 01:45 UT is indicated as a sector marked in grey colour.

survey of the evolution of the measured angle $a k$, again for the continuum emission line at $\sim 70 \mathrm{kHz}$, in the time interval between 01:45 and 02:30 UT. It indicates that the slow drift in $a k$ values on all four spacecraft is dominated by large and rapid angle fluctuations on each satellite, in the range 78 to $98^{\circ}$. This range is not much larger than the $\pm 7^{\circ}$ uncertainty span, but some of it could be real. The large fluctuations are not correlated from one spacecraft to another. This is not in favour of a variability of the angle estimations due to time evolutions in a common source. The fluctuations could perhaps be related to small spatial features encountered along the four ray paths, including magnetopause and magnetosheath traversals. Other studies, when the instrument delivers directivity with a better accuracy, should be conducted to explore various possibilities. In summary, although the source cannot be precisely located in this case, we think that it is probably placed near an irregular equatorial plasmapause surface in the morning sector. In such circumstances, it is possible to locally emit radiation in a direction which is perpendicular to the otherwise time and spatially averaged "smooth" plasmapause surface.

\section{Observations in the vicinity of the sources}

\subsection{Case of 23 April 2002: source finding at low latitude}

Occasionally, Cluster observes stable, discrete continuum elements while crossing the magnetic equator. Figure 6 presents such an event, obtained at small separation, where the maximal distance between spacecraft is $\sim 140 \mathrm{~km}$ in the XY-plane and $\sim 240 \mathrm{~km}$ along Z-axis. The top panel shows for $\mathrm{C} 4$ a dynamic spectrogram of the signal intensity over 
the frequency range where the continuum elements are observed. The middle panel displays in the same way the $a k$ angle values obtained from the direction analysis described in Sect. 2.3. The Whisper instrument is working in its standard operation mode, as during the case event presented before. The frequency separation does not allow the resolution of very fine structures: moreover, direction finding requires acquisition time intervals of about $90 \mathrm{~s}$ for enough sample points to be collected. Despite those limited performances, the direction analysis indicates clearly a systematic evolution of the propagation direction during the pass, similar on all continuum elements. All of them show a similar evolution of the $a k$ value: at about $30^{\circ}$ when first showing up in the Southern Hemisphere, the $a k$ value decreases down to about $-50^{\circ}$, when the emission line fades out.

We have compared the propagation directions for the continuum element of lowest frequency, which is the best defined in this case. Its frequency is increasing from 70 to $72 \mathrm{kHz}$ during the pass and we have selected three different times for performing direction finding. The results are presented in the bottom panel of Fig. 6. They show that:

1. The cone angle spanned by the four directions at a given time is large, up to about $30^{\circ}$. This can be explained by the low accuracy of the determination. Indeed, in addition to the $\pm 7^{\circ}$ uncertainty corresponding to the general scatter in the data (see section 2.3), we must account for the fact that the view angle of a spacecraft over a narrow source varies during the $90 \mathrm{~s}$ necessary for the analysis. As the corresponding displacement in the XY-plane is about $170 \mathrm{~km}$, and as the source is placed at about $1500 \mathrm{~km}$ from the orbit, as deduced from the triangulation, we evaluate the view angle variation to $6.5^{\circ}$, and consider it must be added to the $14^{\circ}$ total span angle due to random noise,

2. Triangulation is nevertheless alright: all directions point roughly toward the same source region. It is quite possible that several small sources account for the apparent single continuum element that Whisper's limited frequency resolution is able to isolate; they are likely part of the same region in space.

We deduce from this successful triangulation that the source position is located over a limited area in the XY-plane, and that it is stable at first approximation during the duration of the observation (about $15 \mathrm{~min}$ ). The derived position, placed between the orbit and the Earth, is in accordance with the assumption of continuum sources located at the equatorial plasmapause. In that particular case, the estimated source position is in the night sector $(\sim 20: 50 \mathrm{LT})$, where the plasmapause is known to be usually well defined, sometimes overlapped by the plasma sheet particle injection boundary. The electron gyrofrequency local to the source can be estimated from the gyrofrequency measured by the sounder at the equator $(7.7 \mathrm{kHz})$ and from compared spacecraft and source geocentric distances. It amounts to $\sim 9.8 \mathrm{kHz}$, much larger than the fine structure frequency separation observed $(\sim 3.2 \mathrm{kHz})$. Several sources of similar brightness are likely in action. They seem to switch off at about the same time, for all spacecraft and all frequencies. Before switch off time, the modulation index is high, over $80 \%$, for the two elements lowest in frequency, whereas it is about $60 \%$ at the higher frequencies.

Details in the switch off times of the continuum elements at $\sim 02: 50$ UT indicate a sequencing versus spacecraft in the order $\mathrm{C} 1, \mathrm{C} 2$ and $\mathrm{C} 4$, then $\mathrm{C} 3$, which, considering the constellation arrangement, corresponds to the traversal of a spatial boundary (latitudinal or azimuthal) in the propagation cone angle, rather than to a time fluctuation in the source. The four spacecraft are too close to each other in this case, and the time resolution is too crude, however, to provide any serious clue to whether it might be a latitudinal or an azimuthal boundary. Concerning a possible boundary in azimuth, one should note that continuum sources are probably drifting according to plasmasphere corotation. Green et al., 2002, for example, describe a case event when the radiation is produced in a narrow emission cone confined to the longitudinal extent of a plasmasphere bite out, observed to corotate with the plasmasphere. In the case of the Cluster event we are discussing (23 April 2002), there is no sign that the emission cone is narrow (the observed range in directivity directions is quite large), but it is still reasonable to consider co-rotation as the first order movement of the source. The corotation of the source region would correspond, as projected in the XY-plane plot of Fig. 6, bottom panel, to a movement in the direction opposite to the spacecraft movement on their orbit. However, the corresponding displacement during the time interval considered, about $500 \mathrm{~km}$, would be smaller than the overall size of the region pointed by the directivity pattern, hence not detectable. Other cases, with larger spacecraft separations can provide more information on this interesting topic.

\subsection{Case of 9 July 2001: a study at medium latitude}

This case event is taken during the approach of the plasmasphere on an inbound leg. The size of the plasmasphere appears stationary on this inbound pass, as all spacecraft encounter the plasmapause (defined here at $\mathrm{F}_{p} \sim 80 \mathrm{kHz}$ ) at similar $\mathrm{L}$ values $(\sim 5.7)$, including $\mathrm{C} 3$, travelling with a time lag of about 35 min behind a trio formed by $\mathrm{C} 1, \mathrm{C} 2$ and $\mathrm{C} 4$. On the outbound leg, two hours later, the plasmasphere appears deeply eroded down to $\mathrm{L} \sim 4.2$ and observations from C3 differ strongly from what is seen on the three other spacecraft. We will come back to this point later.

The frequency-time spectrograms measured inbound by the four Cluster are shown in Fig. 7 (left panel) for a 20-min time interval. They are better understood when compared to the orbital plot shown in the top right panel: the trio $\mathrm{C} 1, \mathrm{C} 2$ and $\mathrm{C} 4$ is placed at proximity of the plasmapause, while $\mathrm{C} 3$ is still located in the high latitude southern plasmatrough. On spectrograms for $\mathrm{C} 1 \mathrm{C} 2$ and $\mathrm{C} 4$, the plasma frequency, precisely identified by the sounder and by a signature (low frequency cut off) in electrostatic emissions, increases smoothly 

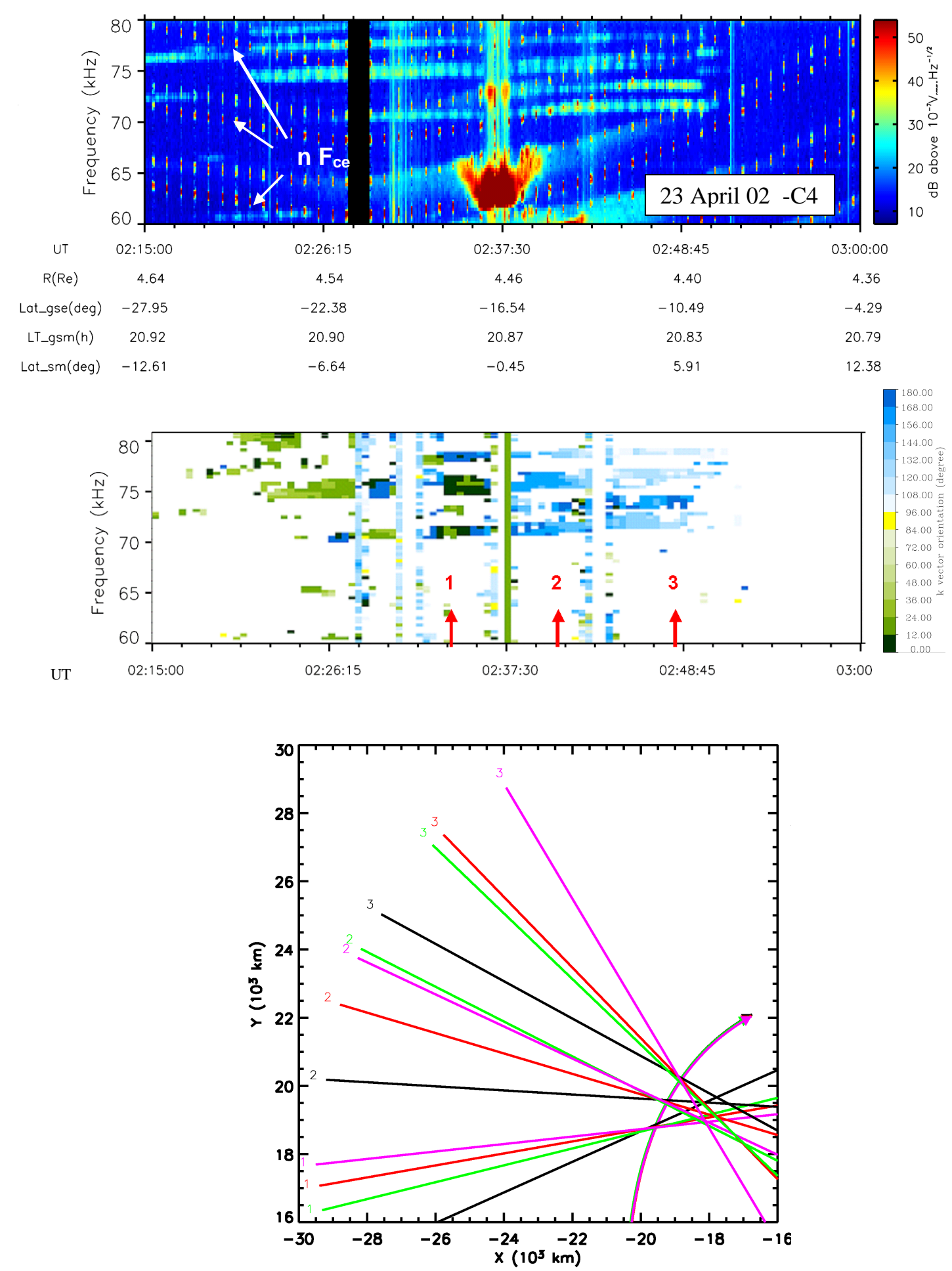

Fig. 6. Triangulation from successive direction findings for the 23 April 2002 event. Top panel: frequency/time spectrogram showing details of continuum elements at Cluster perigee. Resonance pattern triggered by the sounder appear as red points. They are composed in this case of $\mathrm{F}_{c e}$ and $\mathrm{F}_{q}$ lines. Middle panel: spectrogram of the $a k$ angle calculated over the same frequency/time interval, showing the spin modulated continuum elements (green to blue horizontal features). All of them show a similar evolution of the $a k$ value. Bottom panel: orbit and propagation directions projected onto the XY GSE-plane. The continuum element of lowest frequency $(\sim 70 \mathrm{kHz})$ has been chosen for the analysis; directions at three instant (as marked by arrows in the middle panel) are shown for the four spacecraft. Although spanning a large angular domain, they all point to the same region, placed between the orbit and the Earth. 
CLUSTER-WHISPER Spectrogram / JUL 09, 2001 (Day 190)
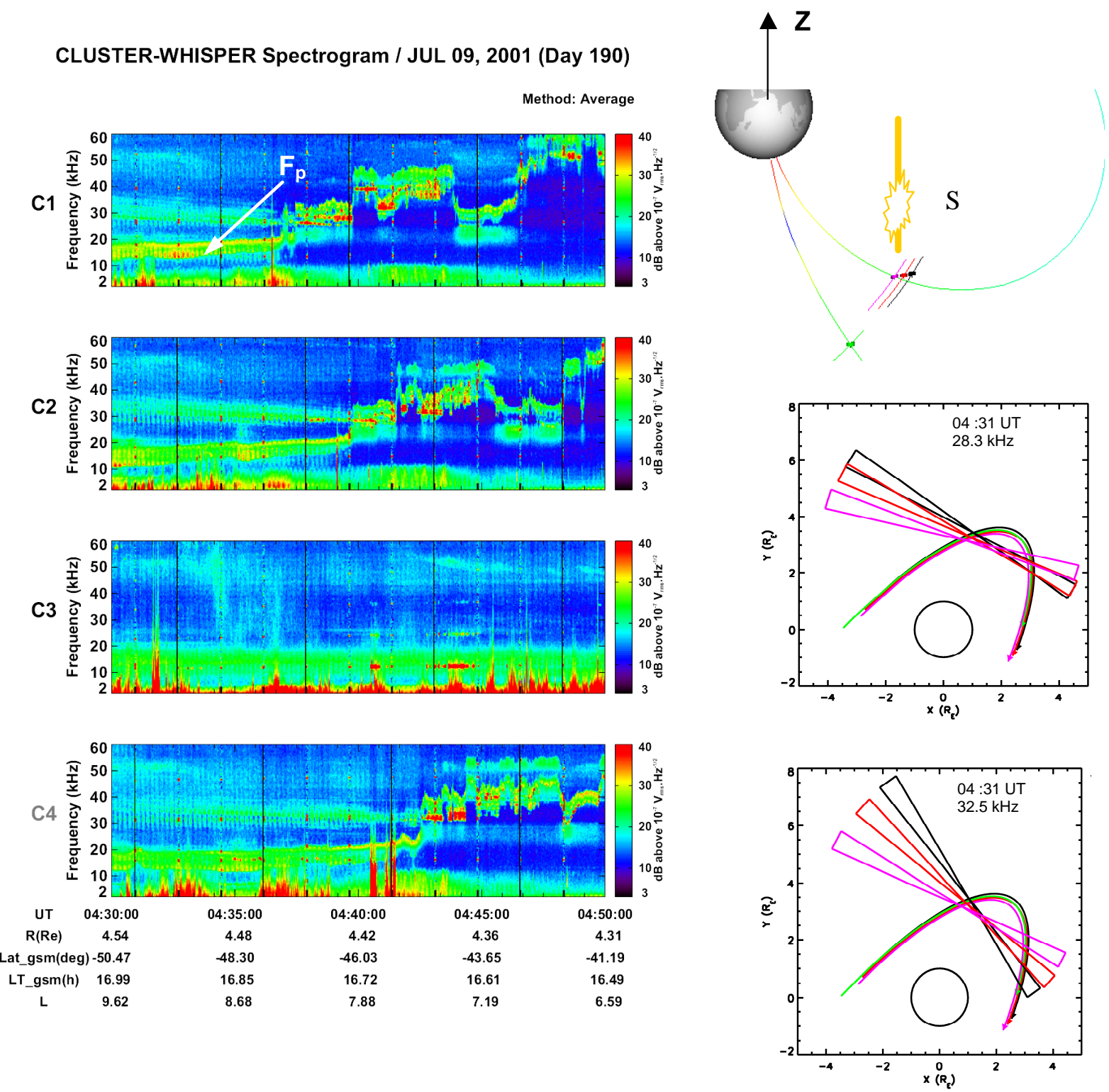

Fig. 7. Direction analysis for the 9 July 2001 event. Left panel: frequency/time spectrograms measured by the four Cluster spacecraft, at the approach of the plasmasphere. Orbital parameters are indicated for the satellite C4. Right, top panel: orbit elements in a meridian plane during the interval 04:25 to 04:55 UT. Spacecraft are heading from Southern to Northern Hemisphere, field lines crossed by C4 (purple symbol) and C3 (green symbol) are drawn at 04:45 UT. The orange segment and patch indicate source positions as discussed in Sect. 4.2. Right, bottom panels: triangulation at two frequencies, same time interval, from Cluster C1, C2 and C4 satellites. Each direction is indicated as the angle spanned over $5^{\circ}$ about the central direction. All point to a source region located earthward from the orbit in the XY GSE-plane shown (orbits and direction angles are projected on to the plane).

from about $12 \mathrm{kHz}$ to $20 \mathrm{kHz}$, until each satellite encounters a region centred at $\mathrm{L} \sim 7.15$, highly structured in density. $\mathrm{C} 3$ encounters similar features when reaching the same $\mathrm{L}$ values later (see Fig. 8, top panel), but, at the time interval of Fig. 7, the density is too low to be measurable by Whisper. The continuum emissions of interest are first seen in the spectrograms of $\mathrm{C} 1, \mathrm{C} 2$ and $\mathrm{C} 4$, a few minutes from the start of the interval shown. They do not display a structured behaviour, but appear as a continuum band between 28 to $36 \mathrm{kHz}$ at 04:30 UT, decreasing smoothly in central frequency as time progresses, until the large irregular density structure encountered blocks the propagation to the spacecraft. Patchy bright emissions are visible inside the density structure, at frequencies apparently in continuity with the banded feature observed outside, but amplified in intensity. Satellite C3 does not observe the continuum band, neither at that time nor later when it reaches the same region (Fig. 8, top panels). Even from a close examination of Fig. 7 spectrograms, it is difficult to make a statement about the connection between a given continuum structure, like the emission line observed on spacecraft $\mathrm{C} 2$ 


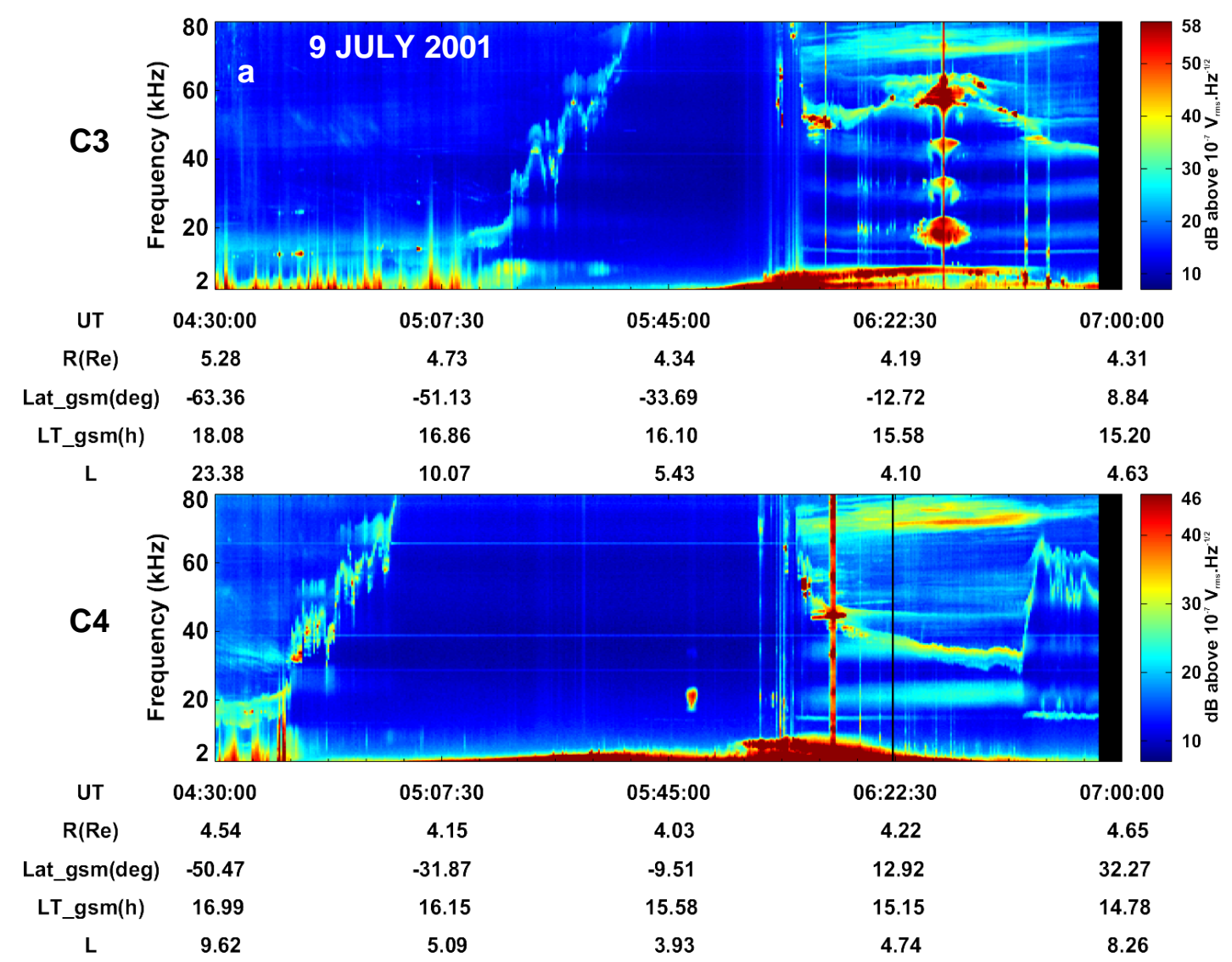

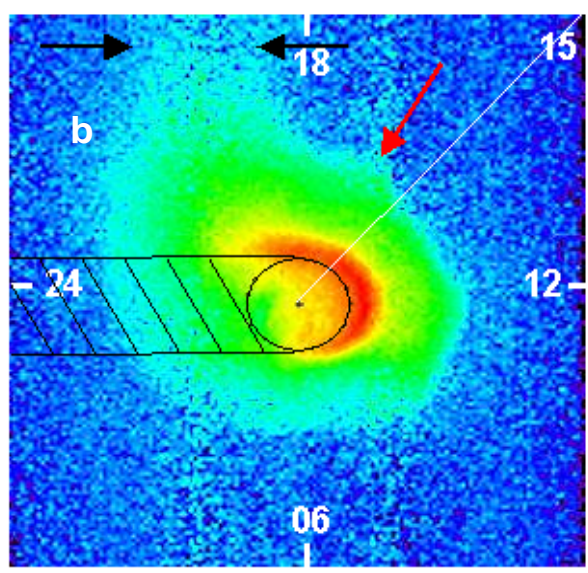

9 JULY 2001 8:18 UT

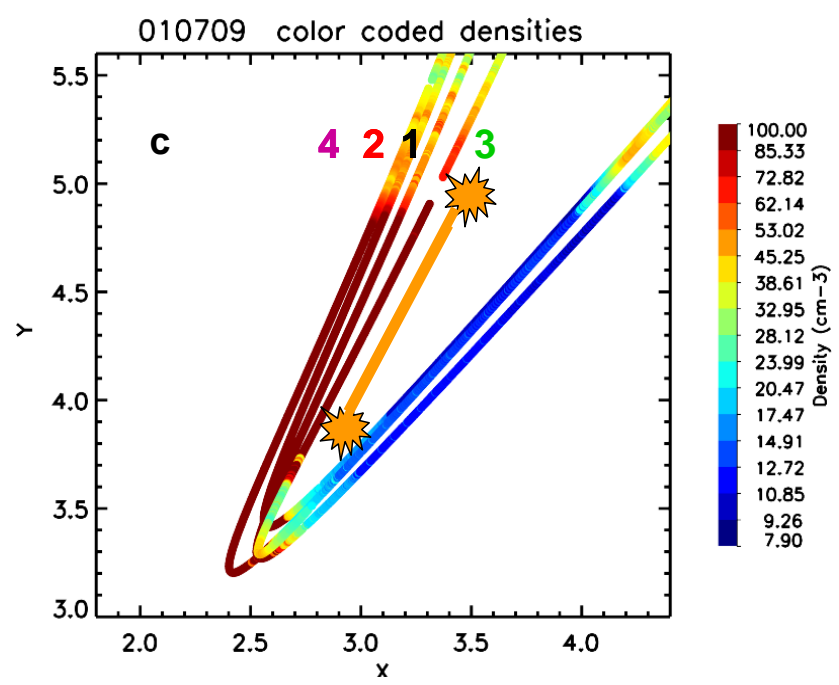

Fig. 8. Global views of the plasmasphere for the event of 9 July 2001. a): Whisper spectrograms for Cluster spacecraft C3 and C4. b): IMAGE/EUV global view of the plasmasphere at 08:18 UT, the red arrow indicates Cluster position; black arrows point to the plume. c): detailed trajectories of the four Cluster spacecraft near perigee, projected along magnetic field lines on to the equatorial plane; measured densities (colour coded) are plotted along the trajectories (upper values capped to $100 \mathrm{~cm}^{-3}$ ). Estimations of the source position are indicated in orange. Numbers at top indicate ordering of spacecraft trajectories.

at 04:38 UT, $28.5 \mathrm{kHz}$, and a given feature observed later, like the bright emission seen on C2 at 04:40 UT, $28 \mathrm{kHz}$, but those are obviously two facets of the same global phenomena. Further examination indicates that some of the bright modulated emissions observed at the end of the interval seem indeed trapped inside local depletions of the density profile (04:39:40 UT and 04:43 UT on C2; 04:41 UT on C1), while others (04:40:10 UT on C2) occur on a density shoulder, hence are not entirely trapped inside a depletion, and could escape. 
Results from direction finding and triangulation analysis at the start of the event (04:31 UT) are shown in the two lower right panels of Fig. 7. The time resolution of the spectra $(1.7 \mathrm{~s})$ allows one direction finding every minute. The satellite $\mathrm{C}$, which does not observe any continuum emission in the time/frequency range of interest, has been excluded. The triangulation is successful, as it is generally later in the pass. We assume that the $180^{\circ}$ ambiguity is resolved by the position of the ray path intersection, and confirmed by the fact that multiple triangulations can be done using more than two spacecraft. To be rigorous, the source position found should be corrected from the displacement caused by refraction effects expected near the source (see, for instance, ray tracing in realistic plasma models in Horne, 1989). Such effects, neglected in a first approximation, would likely place the source slightly closer to the spacecraft than what triangulation indicates, a spacecraft to source distance of $\sim 0.5 \mathrm{R}_{E}$, when projected in the XY GSE-plane. Our triangulation findings indicate thus a source region located on a column at about $3 R_{E}$ from the Z GSE-axis (see sketch on Fig. 7, top right). The column is placed relatively closer to the Earth at $32.5 \mathrm{kHz}$ than at $28.3 \mathrm{kHz}$, as should be expected if the frequency is related to the local density at the source. This $2-\mathrm{D}$ view is a real achievement. It does not provide the full 3-D view, but opens an interesting discussion: where is the source?

The classical view of a source placed both at plasmapause and at the equator is ruled out, considering two evidences. The first evidence is that a source placed at the equator at $3 R_{E}$ would correspond to a propagation direction almost perpendicular to the equatorial plane, i.e. quasi-aligned with the local magnetic field. Such a direction is not expected. In the frame of the linear window conversion theory (Jones, 1982) the beaming angle, i.e. the angle of the radiation vector with the equator, would be of $\sim 45^{\circ}$ (for the estimation $\mathrm{F}_{c e} \sim \mathrm{F}_{p} \sim 30 \mathrm{kHz}$ at the source). Furthermore, left-hand polarized ordinary $(\mathrm{L}-\mathrm{O})$ mode waves are considered in the frame of this theory, and indeed observed (Gurnett et al., 1988). They show quasi-circular polarisation characteristics, and would lead to a very weak modulation index for a wave vector at small angle with spin axis. We observe, on the contrary, significant modulation index values during this event, varying in the 40-90\% range. Non linear generation models (Christiansen et al., 1984; Rönnmark, 1985) are less developed; several wave mode and polarization characteristics have been proposed, but none with a propagation vector along the magnetic field. The second evidence is that the equatorial plasmapause observed on the inbound path is farther off the Earth, by 2 to $3 R_{E}$, than the column derived by the triangulation. Even the compressed plasmapause boundary crossed outbound at $L \sim 4.2$ is placed significantly farther from the Earth than the equatorial foot of the derived column.

The assumption of a source placed at a plasmapause boundary but off the equator is more plausible. Releasing the constraint about source proximity with the equator, other constraints are released as well. The plasmapause boundary at latitudes comparable to the constellation latitude $\left(\sim-35^{\circ}\right)$ is placed at close distance from the spacecraft, in between
Cluster and the Earth, as required by triangulation results. The source to spacecraft propagation path can thus make a small or moderate angle with the spin plane, as required from large modulation index values. Trying to go further along that line, we consider making use of actual modulation index values as a proxy for the out of spin plane propagation direction. Indeed, the modulation index would vary as $\cos (\psi)$ in the case of a circular polarisation, a point source, and a propagation at an angle $\psi$ with spin plane. The finite size of the source, or its equivalent in case of multiple point sources, would reduce the measured modulation. The value of $\psi$ deduced from our simple model can only be overestimated. From the limited analysis performed in this study, and global parameters obtained (source to spacecraft distance and modulation indices averaged over frequency and spacecraft), it can be stated that the propagation direction is at an angle of less than $\sim 40^{\circ}$ from the ecliptic plane, thus that the source region detected by the constellation is probably located at a distance of at least $2.5 R_{E}$ south from the ecliptic plane (up to $3 R_{E}$ for highest modulation values). This corresponds to at least $1.8 R_{E}$ south in SM coordinates. Non equatorial events have been already observed (Morgan and Gurnett, 1991). The multipoint view of Cluster will certainly bring new information about those cases.

Another question to consider is the overall shape of the plasmapause boundary, and the source position with respect to this boundary. It is interesting to note that, as in cases presented above (Figs. 4 to 6), the directions shown in Fig. 7 are far from radial, a behaviour not compatible (in the frame of the linear window theory) with a smooth circular plasmapause surface.

We have gathered in Fig. 8 more information about the plasmasphere shape and dynamics for that particular event. The geomagnetic activity is unsteady during the few hours preceding the event ( $K_{p}$ varying in both directions in the range 1 to 4$)$. The global images of the plasmasphere taken by the Extreme Ultraviolet (EUV) instrument (Sandel et al., 2000) on the IMAGE mission (Burch, 2000) are not available at the precise time interval of our continuum observations. Because of IMAGE high apogee $\left(\sim 7 R_{E}\right)$ and the EUV imager's wide field of view, images acquired near apogee show the structure of the entire plasmasphere. A few hours before the interval (at 00:18 UT), the EUV image (not shown) indicates a regular plasmasphere shape. The EUV image obtained a couple of hours after the interval is shown in Fig. 8b. It indicates the presence of a large plasmaspheric plume in the dusk sector (18:00-20:00 MLT), and of a small bulge in the postnoon sector (16:00 MLT). It must be noted that the positions of the density boundary visible in the EUV image are only indicative of a plasmapause boundary in an L-MLT frame, as line of sight density boundaries are not necessarily projected onto the equatorial plane. In any case, they show that the plasmapause surface in the local time sector of Cluster trajectories (where the small bulge is observed) is not smooth. The density profiles measured by Cluster provide details about the plasmapause position and dynamics at the time of the event. The two spectrograms at the top of 
Fig. 8 indicate a striking difference between conditions encountered at perigee crossing by $\mathrm{C} 4$ at 05:49 UT (high densities, inward from the plasmapause) and at perigee crossing by C3 later (lower densities, outward the plasmapause), although they follow each other on very similar orbits (see Fig. 8c). They actually cross a plasmapause boundary outward at about the same time (06:05 UT), but not at the same MLT, C4 on its outbound leg, C3 still on its inbound leg (it crosses perigee at 06:31 UT). Figure 8c displays density profiles (colour-coded values) for the four Cluster trajectories plotted in an L-MLT frame (each indicated position is the intersection with the GSM equatorial plane of the field line crossed by the spacecraft at a given time of its journey). It shows how MLT decreases from the eastward inbound leg (high densities) to the westward outbound leg (low densities). It is not possible to know if the very different inbound conditions encountered by $\mathrm{C} 3$ and by the 3 other Cluster satellites is the effect of a violent erosion (or compression) of the plasmasphere occurring at about 06:05 UT, or if a large density notch, already present at the time of our continuum event (at 04:30 UT), is moving eastward, possibly at modest speed, so that it catches all spacecraft at about 06:05 UT and remains eastward from $\mathrm{C} 3$. The density increase at the end of the $\mathrm{C} 4$ pass (Fig. 8a) indicates that indeed $\mathrm{C} 1, \mathrm{C} 2$ and $\mathrm{C} 4$ are located inside a density notch between 06:00 and 07:00 UT.

The rough estimation of source location along the column parallel to Z GSE-axis (estimated from modulation index values to belong to the interval 2.5 to $3 R_{E}$, Southern Hemisphere), combined with its position in the XY-plane obtained by triangulation (Fig. 7), allow us (after a projection along a magnetic field line) to place the source in the L-MLT plot of Fig. 8c. The two extreme positions figured correspond to the two extremes of the Z GSE values considered. They are placed inside the density notch crossed by the constellation after 06:05 UT. It is worth noting that Whisper observes in this region of depressed densities, both continuum radiation features over the high end of the Whisper frequency range, and intense electrostatic emissions thought to be primary sources of continuum. Such an intense electrostatic source is crossed by $\mathrm{C} 4$ at $\sim 06: 08 \mathrm{UT}$, saturating the Whisper analyser (vertical red bar). It it located off the equator. Another such source is crossed by $\mathrm{C} 3$ at the equator (at about 06:30 UT). Since continuum radiations observed at $\sim 04: 30$ UT are placed at lower frequency than the various features observed in the density depression, no direct connection can be made. However, we can state that general conditions favourable to continuum generation are encountered precisely where geometrical considerations, derived from measured directivity characteristics, allow us to place the source acting at 04:30 UT.

In conclusion, this limited analysis demonstrates that the desire to find the precise location of continuum sources, even with the help of multi-spacecraft measurements, encounters difficulties due to the genuinely complex and dynamical conditions met. Our best estimation in the event case studied indicates a source placed at the plasmapause, off the equator. To sustain this scenario, density holes would have to be present such as to allow for a free path between the source and observatories. Large irregularities, observed at plasmapause by all Cluster spacecraft, show that indeed the plasmapause is deeply structured in this local time sector and could thus provide the required conditions. The IMAGE view observed later confirms the existence of a structured plasmapause in the MLT sector of interest.

\section{Summary and conclusion}

The non-thermal terrestrial continuum radiation is observed by the four satellites of the Cluster fleet in a variety of magnetospheric regions, including solar wind (not shown), magnetosheath, lobes and inner magnetosphere, in the latter case at close proximity of initial driving sources. The various sizes of the Cluster configuration during the mission phase $(100 \mathrm{~km}$ up to $5000 \mathrm{~km}$, and up to $15000 \mathrm{~km}$ in the future) allow complementary views of a geophysical phenomenon which, although very frequent and already studied from a number of space missions, is still poorly understood. We have chosen in this paper to present the main results we could obtain from a first analysis of four-point observations of the continuum emissions by the Whisper instrument. Whisper is the Cluster instrument which, in the wave consortium WEC (Pedersen et al., 1997), is dedicated to the four-point survey of wave emissions in the frequency range covering the continuum radiation band, here limited to the $2-80 \mathrm{kHz}$ range.

We have chosen a few events representative of the type of information we can obtain from Whisper data alone, presented some of the analysis which is possible using first a single spacecraft, then the complete fleet. Finally, we have discussed the results obtained in each event in the frame of the main knowledge accumulated from previous studies.

The first information we obtain about the continuum is the spectral behaviour of the electric field of the wave and its dynamics. Single spacecraft dynamic spectrograms can be compared, one to another, by visual examination (see Fig. 7). The second type of information is the result of direction finding analysis performed from spin modulation of the spectra. We have presented a description of the technique used and of corresponding performances in direction finding. It appears that the resulting uncertainty on the 2-D directivity angle is quite variable, from $\sim \pm 2^{\circ}$ to $\sim \pm 10^{\circ}$. Directivity angles and modulation indices derived from the direction finding analysis can be visualized as single spacecraft dynamic spectrograms (see upper panels of Fig. 3), which can be discussed individually or by comparison. Small differences from spacecraft to spacecraft have been pointed out by detailed superposed plots (Fig. 3, bottom, and Fig. 4, top).

Our findings derived from directivity investigations using the complete fleet can be summarized as follow:

1. The directivity view demonstrates its full power when used in the vicinity of the sources. We can then apply a 2-D triangulation of the source location. We have shown in one event case (Fig. 7) a successful instantaneous 
triangulation, confirmed by the redundancy offered by having a three-point baseline. The lack of observed continuum from the fourth point illustrates the importance of the 3-D geometry, to be investigated. In another event, a large uncertainty on the directivity angle does not permit direct triangulation. However, the source stability leads to an indirect triangulation, as each spacecraft, travelling on its orbit, forms a triangulation baseline (Fig. 6). In that case, obtained with small spacecraft separation, the redundancy allowed by the four measurements increases the reliability of the estimation.

2. When yielded at a distance of the source, the multispacecraft directivity view offers a potentially interesting estimation, not yet investigated, of the randomness in the directivity measurements due to local factors. It also helps to reduce the estimated angular dimension of the source region. Triangulation at large distance from the Earth, for instance, from the magnetosheath, is yet not applicable, but should be during the final mission phases, where large spacecraft separations are foreseen.

3. Another aspect of the multi-spacecraft directivity view has been briefly discussed in the paper: the analysis of the evolution of directivity angle and modulation index as a function of the spin plane distance to the ecliptic. Such analyses, made in conjunction with the 2-D investigations offered by the multiple baseline, offer a potentially powerful means toward a 3-D remote view of the continuum sources.

The results of geophysical interest obtained from our limited event study are listed below:

1. The directivity characteristics confirm cases of efficient radiation trapping in the lower frequency band, of radiation collimation along the Sun-Earth direction in the medium frequency range, likely from reflection at magnetopause, of the transparency of the magnetosheath boundary in the highest frequency range.

2. In all cases examined, the scenario of a source near the plasmapause is consistent with the observations. Successful triangulation analyses indicate in one event a source in the pre-midnight sector, near the equator; in another event a source off the equator, in the dusk sector. A possible role of wave ducting has to be investigated.

3. Latitudinal differences in directivity properties have been observed; they have still to be explained, and detailed beaming characteristics to be established.

4. Local plasma diagnostics by the sounder help to identify the nature of electrostatic emissions which are related with driving sources of continuum radiation; emissions at upper hybrid frequency might not always be the major driving source.

5. Continuum radiation amplification is observed in conjunction with local density depletions of small size in the magnetosheath (see Sect. 3.2) and plasmasphere (see Sect. 4.2). The full importance of plasma irregularities on radiation properties, including propagation path, has to be established.

6. The role of large size density irregularities at plasmapause on the generation and beaming properties of continuum radiation is an interesting topic to be further addressed. Such studies will benefit from the comparison of Cluster local detailed views with large view imaging from IMAGE when at apogee.

In conclusion, first investigations on continuum radiation properties and on remote sensing of the source regions based on Cluster fleet data are very encouraging. New studies can now be envisaged, both at small scale, for example about the nature of the sources or the role of small density irregularites, and at larger scale, for example about typical beam extensions or other continuum propagation or optical properties. We hope for a renewed interest about the terrestrial continuum emissions that will drive various studies and approaches in the future. A better understanding of their behaviour is necessary if we want for instance to use them as a tool for remote sensing the plasmasphere or the magnetosheath.

Acknowledgements. We are grateful to B. R. Sandel for providing images from the EUV instrument on board the IMAGE spacecraft, and to $\mathrm{M}$. André and the EFW team for sharing data from their CLUSTER instrument. We would like to warmly thank the teams at ESOC (Darmstadt), JSOC and Sheffield (UK) for their constant support of CLUSTER operations. We acknowledge A. Masson and O. Moullard for sharing useful views about density derivation by combining EFW and Whisper data.

Topical Editor T. Pulkkinen thanks J. Green and another referee for their help in evaluating this paper.

\section{References}

Barbosa, D. D.: Low-level VLF and LF radio emissions observed at Earth and Jupiter, Rev. Geophys. Space Phys., 20, 316-334, 1982.

Burch, J. L.: IMAGE mission overview, Space Sci. Rev., 91, 1-14, 2000.

Canu, P., Décréau, P.M.E., Trotignon, J.-G., Rauch, J.-L., Séran, H. C., Fergeau, P., Lévêque, M., Martin, Ph., Sené, F. X., Le Guirriec, E., Alleyne, H., and Yearby, K.: Identification of natural plasma emissions observed close to the plasmapause by the Cluster-Whisper relaxation sounder, Ann. Geophys., 19, 16971709, 2001.

Christiansen, P. J., Etcheto, J., Rönmark, K., and Stenflo, L.: Upper hybrid turbulence as a source of nonthermal continuum radiation, Geophys. Res. Letters, 11, 139-141, 1984.

Darrouzet, F., Décréau, P. M. E., De Keyser, J., Masson, A., Gallagher, D., Santolik, O., Sandel, B. R., Trotignon, J. G., Rauch, J. L. Le Guirriec, E., Canu, P., Sedgemore, F., André, M., and Lemaire, J. F.: Density structures inside the plasmasphere : Cluster observations, Ann. Geophys., 22, 7, 2577-2585, 2004.

Décréau, P. M. E., Fergeau, P., Krasnoselskikh, V., Lévêque, M., Martin, P., Randriamboarison, O., Sené, F. X., Trotignon, J.-G., 
Canu, P., Mögensen, P. B., and Whisper Investigators: WHISPER, a Resonance Sounder and Wave Analyzer: Performances and Perspectives for the CLUSTER mission, Space Science Reviews, 79, 107-136, 1997.

Décréau, P. M. E., Fergeau, P., Krasnoselskikh, V., Le Guirriec, E., Lévêque, M., Martin, P., Randriamboarison, O., Rauch, J.L. Sené, F. X., Séran, H. C., Trotignon, J.-G., Canu, P., Cornilleau, N., de Féraudy, H., Alleyne, H., Yearby, K., Mögensen, P. B., Gustafsson, G., André, M., Gurnett, D.A, Darrouzet, F., Lemaire, J., Harvey, C. C., Travnicek, P., and Whisper Experimenters: Early results from the Whisper instrument on CLUSTER: an overview, Ann. Geophys., 19, 1241-1258, 2001.

Escoubet, C. P., Fehringer, M., and Goldstein, M.: The Cluster mission, Ann. Geophys., 19, 1197-1200, 2001.

Etcheto, J., Christiansen, P. J., Gough, M. P., and Trotignon, J.-G.: Terrestrial continuum radiation observations with GEOS-1 and ISEE-1, Geophys. Res. Letters, 9, 1239-1242, 1982.

Filbert, P. C. and Kellogg, P. J.: Observations of low-frequency radio emissions in the Earth's magnetosphere, J. Geophys. Res., 94, 8867-8885, 1989.

Gough, M. P., Christiansen, P. J., Martelli, G., and Gershuny, E. J.: Interaction of electrostatic waves with warm electrons at the geomagnetic equator, Nature, 279, 515, 1979.

Gough, M. P.: Non-thermal Continuum emissions associated with electron injections: remote plasmapause sounding, Planet. Space Sci., 30, 657-668, 1982.

Green, J. L. and Boardsen, S. A.: Confinement of nonthermal continuum radiation at low latitudes, J. Geophys. Res., 104, $10307-$ 10316, 1999.

Green, J. L., Sandel, B. R., Fung, S. F., Gallagher, D. L. and Reinish, B. W.,: On the origin of kilometric radiation, J. Geophys. Res., 107 (A7), 1105, doi:10.1029/2001JA000193, 2002.

Gurnett, D. A.: The Earth as a Radio source: The Nonthermal Continuum, J. Geophys. Res., 80, 2751-2763, 1975.

Gurnett, D. A., Calvert, W., Huff, R. L., Jones, D., and Sugiura, M.: The polarization of escaping terrestrial continuum radiation, J. Geophys. Res., 93, 12 817-12 825, 1988.

Gustafsson, G., Boström, R., Holback, B., Holmgren, G., Lundgren, A., Stasiewicz, K., Ahlen, L., Mozer, F. S., Pankow, D., Harvey, P., Berg, P., Ulrich, R., Pedersen, A., Schmidt, R., Butler, A., Fransen, A. W. C., Klinge, D., Thomsen, M., Fälthammar, C. G., Lindqvist, P.-A., Christenson, S., Holtet, J., Lybekk, B., Sten, T.A., Tanskanen, P., Lappalainen, K., and Wygant, J.: The electric field and wave experiment for the Cluster mission, Space Sci. Rev., 79, 137-156, 1997.

Horne, R. B.: Path-integrated growth of electrostatic waves: the generation of terrestrial myriametric radiation, J. Geophys. Res., 94, 8895-8909, 1989.
Jones, D.: Terrestrial myriametric radiation from the Earth's plasmapause, Planet. Space Sci., 30, 399-410, 1982.

Kasaba, Y., Matsumoto, H., Anderson, R. R., Bougeret, J.-L., Kaiser, M. L., Wu, X. Y., and Nagano, I.: Remote sensing of the plasmapause during substorms : Geotail observation of nonthermal continuum enhancement, J. Geophys. Res., 103, 20389 $20405,1998$.

Kurth, W. S., Gurnett, D. A., and Anderson, R. R.: Escaping nonthermal continuum radiation, J. Geophys. Res., 86, 5519-5531, 1981.

Morgan, D. D. and Gurnett, D. A.: The source location and beaming of terrestrial continuum radiation, J. Geophys. Res., 96, 95959613, 1991.

Nagano, I., Wu, X.-Y., Tanako, H., Yagitani, S., Matsumoto, H., Hashimoto, K., and Kasaba, Y.: Remote sensing the magnetosheath by the spin modulation of terrestrial continuum radiation, J. Geophys. Res., 108 (A6), 1224, doi:10.1029/2002JA009691, 2003.

Pedersen, A., Cornilleau-Wherlin, N., De La Porte, B., Roux, A., Bouabdellah, A., Décréau, P. M. E., Lefeuvre, F. , Sené, F. X., Gurnett, D., Huff, R., Gustafsson, G., Holmgren, G., Woolliscroft, L., Alleyne, H. St. C. , Thompson J. A., and Davies, P. N. H.: The Wave Experiment Consortium (WEC), Space Sci. Rev., 79, 157-193, 1997.

Rönnmark, K.: Generation of magnetospheric radiation by decay of Bernstein Waves, Geophys. Res. Letters, 12, 639-642, 1985.

Sandel, B. R., Broadfoot, A. L., Curtis, C. C., King, R. A., Stone, T. C., Hill, R. H., Chen, J., Siegmund, O. H. W., Raffanti, R., Allred, D. D., Turley, R. S., and Gallagher, D. L.: The extreme ultraviolet imager investigation for the IMAGE mission, Space Sci. Rev., 91, 197-242, 2000.

Stasiewicz, K.: OVT Visualization Tool-2 for CLUSTER, User Guide, Copyright (C2000 by the OVT team, version 2.0, http: //ovt.irfu.se, 2001.

Steinberg, J.-L., Hoang, S. and Thomsen, M. F.: Observations of the Earth's Continuum radiation in the distant magnetotail with ISEE 3, J. Geophys. Res., 95, 20 781-20 791, 1990.

Trotignon, J.-G., Décréau, P. M. E., Rauch, J.-L., Randriamboarison, O., Krasnoselskikh, V., Canu, P., Alleyne, H., Yearby, K., Le Guirriec, E., Séran, H. C., Sené, F.-X., Martin, Ph., Lévêque ,M., and Fergeau, P.: How to determine the thermal electron density and the magnetic strength from the CLUSTER/WHISPER observations around the Earth, Ann. Geophys., 19, 1711-1720, 2001.

Trotignon, J.-G., Décréau, P. M. E., Rauch, J. L., Le Guirriec, E., Canu, P., and Darrouzet, F.: The whisper relaxation sounder onboard cluster: A powerful tool for space plasma diagnosis around the Earth, Cosmic Research, 41 (4), 369, 2003. 